ARTICLE

\title{
$\Delta$ Np63 regulates a common landscape of enhancer associated genes in non-small cell lung cancer
}

Marco Napoli (10 1,2,6, Sarah J. Wu 1,2,6, Bethanie L. Gore ${ }^{1,2}$, Hussein A. Abbas (1) 1,2, Kyubum Lee ${ }^{3}$, Rahul Checker ${ }^{1,2}$, Shilpa Dhar ${ }^{4}$, Kimal Rajapakshe ${ }^{5}$, Aik Choon Tan ${ }^{3}$, Min Gyu Lee ${ }^{4 凶}$, Cristian Coarfa (i) ${ }^{5 凶} \&$ Elsa R. Flores (iD 1,2凶

Distinct lung stem cells give rise to lung adenocarcinoma (LUAD) and squamous cell carcinoma (LUSC). $\Delta \mathrm{Np} 63$, the p53 family member and p63 isoform, guides the maturation of these stem cells through the regulation of their self-renewal and terminal differentiation; however, the underlying mechanistic role regulated by $\Delta \mathrm{Np} 63$ in lung cancer development has remained elusive. By utilizing a $\Delta$ Np63-specific conditional knockout mouse model and xenograft models of LUAD and LUSC, we found that $\triangle$ Np63 promotes non-small cell lung cancer by maintaining the lung stem cells necessary for lung cancer cell initiation and progression in quiescence. ChIP-seq analysis of lung basal cells, alveolar type 2 (AT2) cells, and LUAD reveals robust $\triangle N$ p63 regulation of a common landscape of enhancers of cell identity genes. Importantly, one of these genes, BCL9L, is among the enhancer associated genes regulated by $\triangle N p 63$ in Kras-driven LUAD and mediates the oncogenic effects of $\triangle N p 63$ in both LUAD and LUSC. Accordingly, high BCL9L levels correlate with poor prognosis in LUAD patients. Taken together, our findings provide a unifying oncogenic role for $\triangle \mathrm{Np} 63$ in both LUAD and LUSC through the regulation of a common landscape of enhancer associated genes.

\footnotetext{
${ }^{1}$ Department of Molecular Oncology, H. Lee Moffitt Cancer Center and Research Institute, Tampa, FL, USA. ${ }^{2}$ Cancer Biology and Evolution Program, H. Lee Moffitt Cancer Center and Research Institute, Tampa, FL, USA. ${ }^{3}$ Department of Biostatistics and Bioinformatics, H. Lee Moffitt Cancer Center and Research Institute, Tampa, FL, USA. ${ }^{4}$ Department of Molecular and Cellular Oncology, University of Texas MD Anderson Cancer Center, Houston, TX, USA.

${ }^{5}$ Department of Molecular and Cell Biology, Baylor College of Medicine, Houston, TX, USA. ${ }^{6}$ These authors contributed equally: Marco Napoli, Sarah J. Wu.

凶email: mglee@mdanderson.org; coarfa@bcm.edu; elsa.flores@moffitt.org
} 
ung cancer is the number one cause of cancer mortality worldwide and non-small-cell lung cancer (NSCLC) comprises $85 \%$ of all lung cancers, with adenocarcinoma (LUAD) and squamous cell carcinoma (LUSC) being the most common subtypes. The poor prognosis and lack of effective treatment of lung cancer identify the need both to better understand the molecular mechanisms underlying its progression and to develop new therapeutic modalities. Due to similarities between tumour cells and stem cells in both signalling pathways and self-renewal abilities, cancer may originate from transformed stem cells ${ }^{1}$. This hypothesis has been applied to the progenitor cell populations of the lung, including basal cells, alveolar type 2 (AT2) cells and bronchioalveolar stem cells (BASCs), which are involved in the regeneration of distinct regions of the lung ${ }^{2}$. The basal cells of the trachea differentiate into ciliated and goblet cells ${ }^{3}$ and are hypothesised to be the cell of origin for LUSC, since both populations express similar histological markers, such as p63 and keratin $5(\mathrm{Krt5})^{4}$. AT2 cells and BASCs maintain the distal lung alveolar epithelium and can initiate $\operatorname{LUAD}^{5,6}$.

The transcription factor p63 is a member of the p53 family expressed in the basal cells of the lung ${ }^{7}$ and is a master regulator of stem cell maintenance and differentiation of all epithelial tissues $^{8-12}$. The characterisation of the role of p63 in the lung epithelium is complex; and, prior to this study, had yet to be elucidated. There are two N-terminal isoforms, TAp63 and $\Delta \mathrm{Np} 63$, with distinct regulatory roles in stem cell populations in the skin: TAp63 regulates self-renewal of skin-derived precursors (SKPs) in the dermal papilla ${ }^{11}$, while $\Delta \mathrm{Np} 63$ performs this function in epidermal basal cells ${ }^{12}$. Similarly, the p63 isoforms exert diverse functions in cancer. While TAp63 serves as a tumour and metastasis suppressor in multiple tumour types ${ }^{13,14}$, we and others have shown that $\Delta \mathrm{Np} 63$ functions as an oncogene by inhibiting the functions of p53, TAp63 and TAp738,15. Importantly, we showed that $\mathrm{p} 53$ deficient tumours require $\Delta \mathrm{Np} 63$ for their maintenance and we have also identified novel ways to therapeutically target p53 deficient and mutant cancers through manipulation of $\Delta \mathrm{Np} 63^{15-17}$.

In lung cancer, $\Delta \mathrm{Np} 63$ is used in the diagnosis of LUSC $^{18}$ and was identified as a significantly altered gene in $44 \%$ of primary LUSCs by the Cancer Genome Atlas (TCGA) ${ }^{19} . \Delta \mathrm{Np} 63$ has been demonstrated to be oncogenic in cutaneous SCC, which shares molecular characteristics and p63 expression patterns with LUSC ${ }^{20}$. The role of p63 in the distal lung has not been characterised to date because of its low expression; however, $\mathrm{p} 63^{+} / \mathrm{Krt}^{+}$distal airway stem cells have been reported to regenerate distal lung after damage with influenza virus ${ }^{21}$, thus indicating an important role for p63 in the distal lung. Furthermore, we found that the $\Delta \mathrm{Np} 63$ transcriptional signatures generated from either keratinocytes or LUSC and LUAD identified $\triangle \mathrm{Np} 63$ as an oncogene in both NSCLC subtypes ${ }^{13}$. Taken together, there is a critical need to understand the contribution of $\Delta \mathrm{Np} 63$ in stem cells of the lung and how it may impact the initiation and progression of non-small cell lung cancer.

Using xenograft models of LUSC as well as the Kras-driven model of LUAD ( $\left.\mathrm{Kras}^{\mathrm{LSL}-\mathrm{G} 12 \mathrm{D} /+}\right)^{22}$ intercrossed with mice carrying conditionally deleted $\Delta N p 63$, we demonstrate that $\Delta \mathrm{Np} 63$ is required for the maintenance of progenitors of NSCLC. By analysing the role of $\triangle N p 63$ in AT2 and basal cells, respective cells of origin for these tumour types, we found that $\Delta \mathrm{Np} 63$ is required for the self-renewal and maintenance of these cells and does so through the regulation of a common landscape of enhancerassociated genes. This landscape is also maintained in Krasdriven LUAD and includes BCL9L, a known oncogene $e^{23-25}$ with expression levels that are prognostic in LUAD patients. Taken together, our findings unveil a crucial role for $\Delta \mathrm{Np} 63$ in driving a common oncogenic transcriptional programme essential for the formation and progression of both LUAD and LUSC, and identify BCL9L as an important mediator of the oncogenic effects of $\triangle \mathrm{Np63}$ in both NSCLC subtypes.

\section{Results}

$\Delta \mathrm{Np} 63$ promotes tumour initiation and progression in nonsmall cell lung cancer. While the contribution of $\Delta \mathrm{Np} 63$ in lung development has been described in refs. ${ }^{26,27}$, its role in lung tumorigenesis is not well understood. In particular, little is known about the role of $\triangle \mathrm{Np} 63$ in LUAD, primarily due to its low expression in this tumour type $\mathrm{e}^{28}$. To investigate the functions of $\triangle \mathrm{Np63}$ in LUAD, we crossed the Kras-driven LUAD mouse model $\left(\mathrm{Kras}^{L S L-G 12 D /+}\right)^{22}$ to the $\Delta \mathrm{Np} 63$ conditional knockout mouse model $\left(\Delta N p 63^{f l f l}\right)^{12}$. The recombination was induced through the administration of intratracheal adenoviral Crerecombinase ${ }^{29}$, which led to a robust recombination efficiency in the trachea and lung parenchyma assessed by a switch from tdTomato (red) to GFP (green) expressed by the ROSA allele ${ }^{30}$. Twenty weeks postinfection with adenoviral Cre, we assessed a greater than $90 \%$ recombination in the trachea and distal lung assessed by GFP expression from the ROSA allele (Supplementary Fig. 1a). We found that $\mathrm{Kras}^{\mathrm{G} 12 \mathrm{D} /+}$ mice formed lung adenomas and a few LUAD as previously reported ${ }^{22}$ (Fig. 1a). In contrast, lungs from $\Delta N p 63^{\Delta / \Delta} ; \mathrm{Kras}^{\mathrm{G} 12 \mathrm{D} /+}$ mice had almost no scorable adenomas or LUAD formation (Fig. 1a). Examination and grading of the lung lesions ${ }^{22}$ revealed a twofold reduction in atypical adenomatous hyperplastic (AAH) lesions, and a fivefold reduction in grade 1 and grade $2+$ in $\Delta N p 63^{\Delta / \Delta} ; \mathrm{Kras}^{G 12 D /+}$ mice compared to $\mathrm{Kras}^{\mathrm{G} 12 \mathrm{D} /+}$ mice (Fig. 1b). Tumours from $\mathrm{Kras}^{\mathrm{G} 12 \mathrm{D} /+}$ mice stained positively yet heterogeneously for $\Delta \mathrm{Np} 63$ (Fig. $1 \mathrm{c}$ and Supplementary Fig. 1b, i), indicating that $\Delta \mathrm{Np} 63$ is indeed expressed in Kras-driven LUAD.

Given the well-established role of $\Delta \mathrm{Np} 63$ in stem cell maintenance in various epithelial tissues ${ }^{9,11,21,31,32}$, we examined the role of $\Delta \mathrm{Np} 63$ in the maintenance of lung stem cells. LUAD has been shown to originate from distal lung stem cells that express SPC or both CCSP and SPC ${ }^{5,6}$. Therefore, we performed immunofluorescence in adenomas and adenocarcinomas derived from $K r a s^{G 12 D /+}$ and $\Delta N p 63^{\Delta / \Delta} ; K_{r a s}{ }^{G 12 D /+}$ mice using the distal lung stem cell markers SPC and CCSP. Notably, we found that $\Delta N p 63^{\Delta / \Delta} ; \mathrm{Kras}^{G 12 D /+}$ tumours had a $50 \%$ reduction in $\mathrm{CCSP}^{+} /$ $\mathrm{SPC}^{+}$cells compared to tumours from Kras ${ }^{G 12 D /+}$ mice, suggesting that $\Delta \mathrm{Np} 63$ serves to maintain the proliferation of distal lung stem cell populations (Fig. 1d). To assess whether $\triangle$ Np63 plays a similar oncogenic role in human LUAD, we performed soft agar assays in three LUAD cell lines expressing Kras $^{G 12 D}$ (H1944, H358 and H2009) to assess anchorageindependent growth as a surrogate for transformation. Downregulation of $\triangle N p 63$ decreased colony formation ability in these three LUAD cell lines compared to the respective control cells (Fig. 1e and Supplementary Fig. 1j). Altogether, these data indicate that LUAD depends on $\triangle \mathrm{Np} 63$ for their formation and progression.

We next evaluated the oncogenic functions of $\Delta \mathrm{Np} 63$ in the context of lung squamous cell carcinoma (LUSC) by knocking down $\Delta N p 63$ in two human LUSC cell lines, H520 and H2170, and performing soft agar assays. Knockdown of $\Delta N p 63$ decreased the ability of $\mathrm{H} 520$ and $\mathrm{H} 2170$ cell lines to form colonies by $50 \%$ compared to colonies from the respective control cell lines (Fig. 1f, g and Supplementary Fig. 1k). Consistent with these in vitro results, knockdown of $\triangle N p 63$ resulted in a two to fivefold reduction in the in vivo tumour formation of the H520 and $\mathrm{H} 2170$ cell lines in xenograft mouse models (Fig. 1h, i). Histological analysis of tumours from xenografts indicated that 
a

$\operatorname{Kras}^{G 12 D /+} \quad \Delta N p 63^{\Delta / \Delta} ; \operatorname{Kras}^{G 12 D /+}$
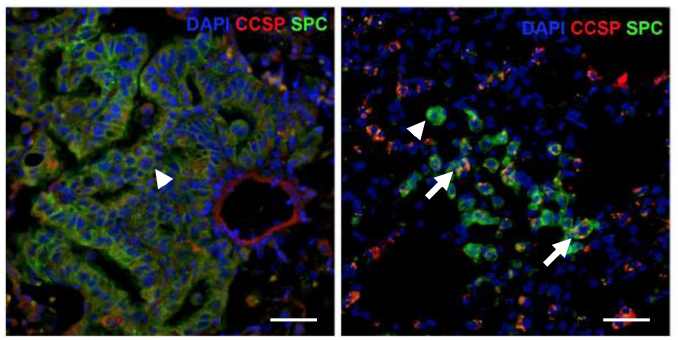

f

LUSC

H520 colony formation

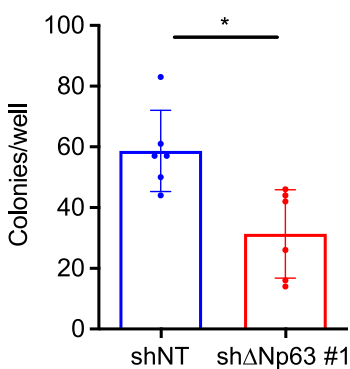

g

$\mathrm{H} 2170$ colony formation
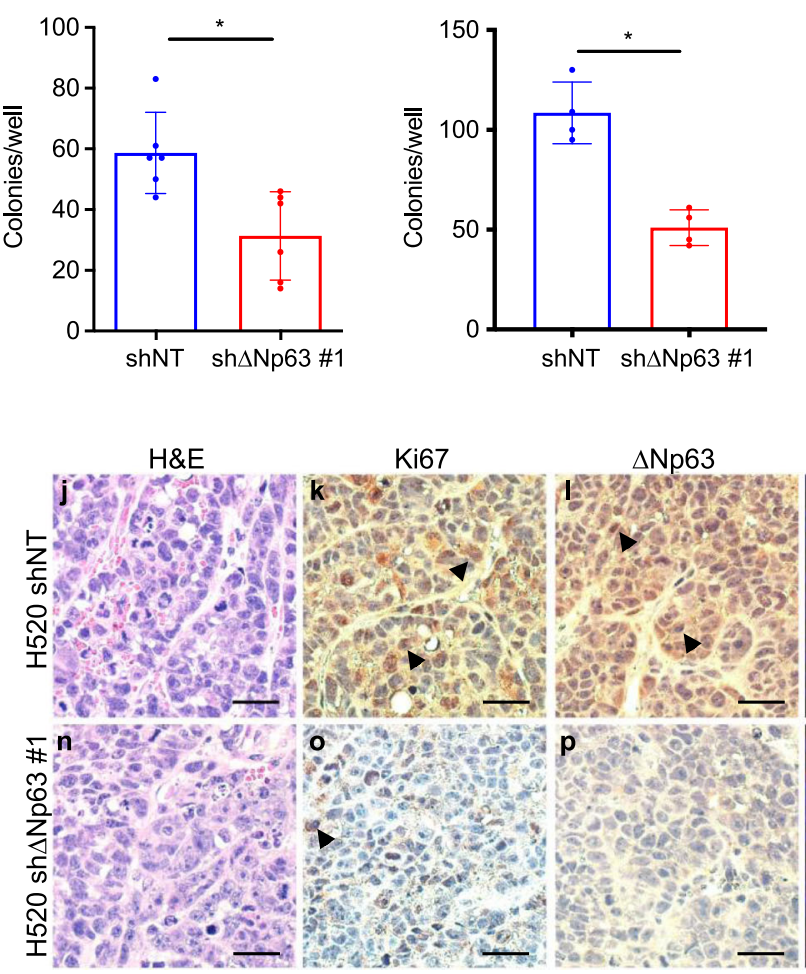

downregulation of $\Delta N p 63$ caused a threefold decrease in proliferation, as assessed by $\mathrm{Ki67}$, and a 20 -fold increase in apoptosis, as assessed by cleaved caspase 3, compared to LUSC tumours expressing $\triangle N p 63$ (Fig. $1 \mathrm{j}-\mathrm{r}$ ). Our laboratory has recently identified a keratinocyte-derived signature of $\Delta \mathrm{Np} 63$ regulated genes and an oncogenic $\Delta \mathrm{Np} 63$-driven transcriptional signature in the context of LUSC and LUAD ${ }^{13}$. These $\triangle \mathrm{Np} 63$ controlled transcriptional programmes support the role of $\Delta \mathrm{Np} 63$ as an oncogene in LUSC, as $\Delta$ Np63 is highly amplified in LUSC ${ }^{19}$

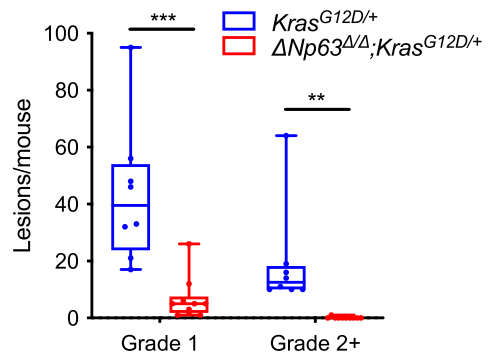

C

e

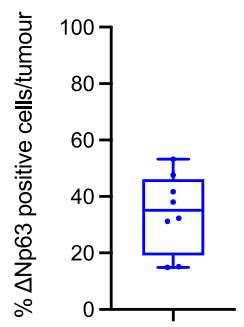

Kras $^{G 12 D /+}$

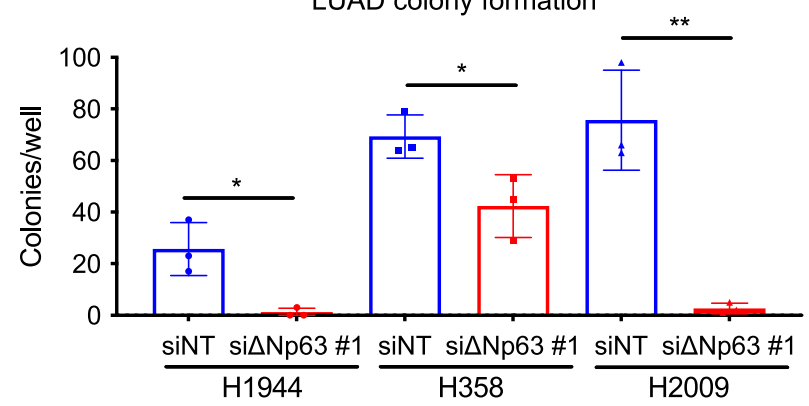

h

LUSC

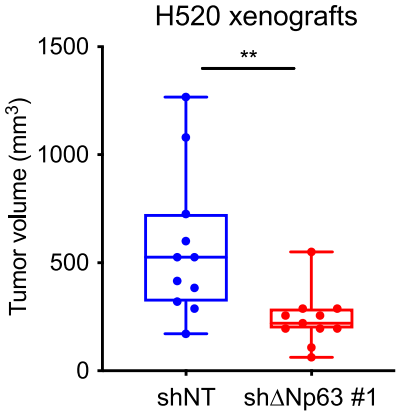

i LUSC

$\mathrm{H} 2170$ xenografts

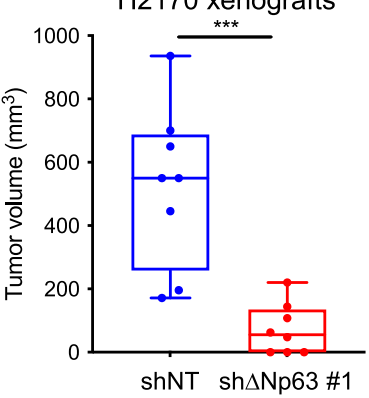

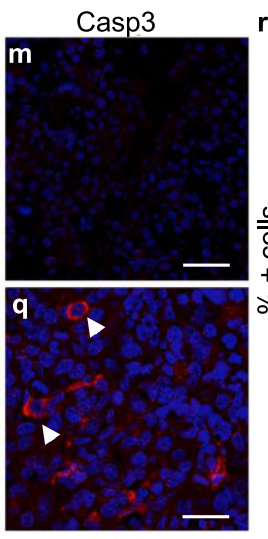

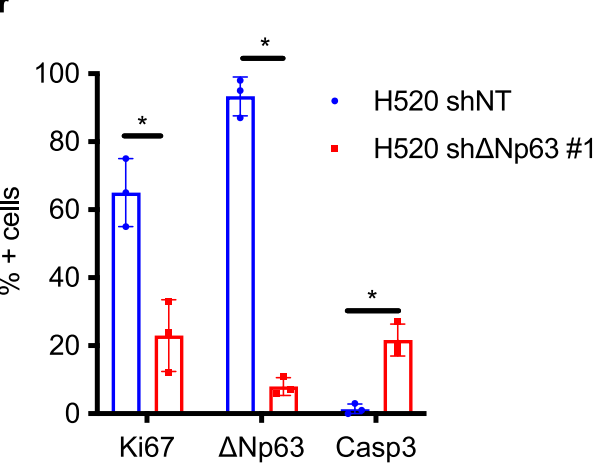

and has been utilised as a positive diagnostic marker for LUSC ${ }^{18}$. Our data with the $\mathrm{H} 520$ and $\mathrm{H} 2170$ cell lines and xenograft models further support the oncogenic role of $\Delta \mathrm{Np} 63$ in the context of LUSC, indicating that LUSC cells depend on $\triangle \mathrm{Np} 63$ for their anchorage-independent growth and xenograft LUSC formation.

Taken together, our data provide evidence that $\Delta \mathrm{Np} 63$ is required for the formation and maintenance of both LUSC and LUAD. 
Fig. $1 \Delta$ Np63 promotes tumour initiation and progression in non-small cell lung cancer. a Representative H\&E stained cross-sections of lung lobes

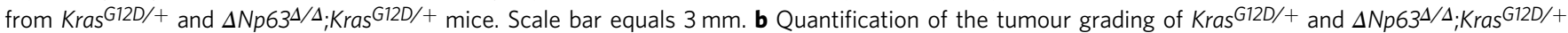
LUAD. AAH atypical adenomatous hyperplasia. LUAD are divided into grade 1 and grade $2+. n=8$ mice, ${ }^{\star \star}$ vs. Kras ${ }^{G 12 D /+, P<0.001,{ }^{\star \star \star}}$ vs. Kras ${ }^{G 12 D /+}$, $P<0.0001$, two-tailed Student's t-test. c Representative image of $\mathrm{IHC}$ for $\Delta \mathrm{Np} 63$ in lung lesions from Kras ${ }^{\mathrm{G} 12 \mathrm{D} /+}$ mice. Scale bar equals $100 \mu \mathrm{m}$. d Representative staining for CCSP and SPC in LUAD from Kras ${ }^{G 12 D /+}$ and $\Delta \mathrm{Np} 63^{\Delta / \Delta ; K r a s}{ }^{G 12 D /+}$ mice. Arrow for a single marker, arrowhead for a double marker. Scale bar equals $100 \mu \mathrm{m}$. e Quantification of colony formation efficiency in soft agar of the indicated LUAD cell lines transfected with the indicated siRNAs. Data are mean $\pm S D, n=3,{ }^{*}$ vs. siNT, $P<0.05,{ }^{\star \star}$ vs. siNT, $P<0.001$, two-tailed Student's $t$-test. $\mathbf{f}, \mathbf{g}$ Quantification of colony formation efficiency in soft agar of the indicated LUSC cell lines infected with the indicated shRNAs. Data are mean $\pm S D, n=6(\mathbf{f})$ and $n=4(\mathbf{g})$, ${ }^{\star} v s$. shNT, $P<0.05$, two-tailed Student's $t$-test. $\mathbf{h}$, $\mathbf{i}$ Tumour volume quantification of xenograft tumours derived from the same cells as in (f, $\mathbf{g}) . n=11(\mathbf{h})$ and $n=8(\mathbf{i})$, ${ }^{\star \star}$ vs. shNT, $P<0.001,{ }^{\star \star \star}$ vs. shNT, $P<0.0001$, two-tailed Student's t-test. $\mathbf{j}-\mathbf{q}$ Representative images of the xenograft tumours described in $\mathbf{h}$ showing H\&E stained cross-sections ( $\mathbf{n}$ ), and staining for Ki67 ( $\mathbf{k}$ and $\mathbf{0}), \Delta \mathrm{Np63}(\mathbf{I}$ and $\mathbf{p}$ ), and cleaved caspase 3 ( $\mathbf{m}$ and $\mathbf{q})$. Scale bar equals $100 \mu \mathrm{m}$. $\mathbf{r}$ Quantification of the stainings shown in $\mathbf{k}-\mathbf{m}$ and $\mathbf{o}-\mathbf{q}$. Data were mean $\pm S D, n=11$, ${ }^{\star} v s$. shNT, $P<0.05$, two-tailed Student's $t$-test. All boxplots represent the individual data points, median and whiskers (min to max method). Source data are provided as a Source Data file.

In vivo $\Delta N p 63$ ablation in the tracheal epithelium results in acute increased proliferation leading to long-term depletion of Krt5 + basal cells. $\triangle \mathrm{Np} 63$ is highly expressed in the basal cells of the trachea, a cell type shown to be the cell of origin for LUSC ${ }^{33}$. To determine whether $\Delta \mathrm{Np} 63$ serves to maintain LUSC through the regulation of tracheal basal stem cells, we utilised $\Delta N p 63^{f l / f l}$ mice crossed with the Rosa reporter mouse ${ }^{30}$ to enable identification of cells that have undergone cre-recombination. To specifically ablate $\Delta N p 63$ in tracheal and lung epithelium, we performed an intratracheal instillation of $\Delta N p 63^{f l / f l} ; R_{0 s} a^{M / M}$ mice with adenoviral cre-recombinase with adenoviral empty vector serving as a negative control. Histological analysis of the trachea and lung tissues of the mice was performed at two timepoints, 1 month and 3 months postinfection. At 1 -month postinfection, quantification of $\mathrm{H} \& \mathrm{E}$ stained sections of $\Delta N p 63^{\Delta / \Delta} ; \operatorname{Ros}^{\Delta / \Delta}$ tracheal epithelium showed a fivefold increase of basal cells within the stratified epithelium (SE) and a fourfold increase of epithelial separation (ES) compared to the $\Delta N p 63^{f l f l} ; R o s a^{M / M}$ control (Fig. 2a, b). Staining for the basal marker keratin 5 (Krt5) revealed that the composition of the stratified epithelium in $\Delta N p 63^{\Delta / \Delta} ; \operatorname{Ros} a^{\Delta / \Delta}$ tracheal epithelium primarily contained Krt5 + basal cells, whereas the $\Delta N p 63^{f l / f l}$; $\operatorname{Rosa}^{M / M}$ control maintained a single basal layer of Krt5+ cells (Fig. 2c, d). Krt5 + cells in $\Delta N p 63^{\Delta / \Delta} ; R_{0 s} a^{\Delta / \Delta}$ tracheas were also fivefold more proliferative as measured by Ki67 staining compared to control (Fig. 2e, f). Intriguingly, this increased proliferation was associated with a strong increase in apoptosis. Indeed, up to $60 \%$ of cells were cleaved caspase 3 positive in the $\Delta N p 63^{\Delta / \Delta} ; R_{0 s a^{\Delta / \Delta}}$ mice compared to almost no cleaved caspase 3 staining in their wildtype counterparts $\left(\Delta N p 63^{f l f l} ;\right.$ Ros $\left.a^{M / M}\right)$ (Fig. $\left.2 \mathrm{~g}, \mathrm{~h}\right)$, suggesting that these cells may undergo depletion or exhaustion over time. Therefore, we assessed the trachea and lung tissues at 3 months postinfection. At that timepoint, the majority of the $\Delta N p 63^{\Delta / \Delta}$; $\operatorname{Rosa}^{\Delta / \Delta}$ tracheal epithelium exhibited a hypoplastic phenotype (HE) compared to the pseudostratified columnar appearance of the $\Delta N p 63^{f l / f l} ; \operatorname{Rosa}^{M / M}$ epithelium (Fig. $2 \mathrm{i}, \mathrm{j}$ ), indicating significant depletion in tracheal epithelial cells lacking $\Delta N p 63$. Krt5 staining of $\Delta N p 63^{\Delta / \Delta} ; \operatorname{Ros}^{\Delta / \Delta}$ tracheal epithelium showed little to no Krt5+ basal cells (Fig. $2 \mathrm{k}, \mathrm{l}$ ), further indicating a depletion or exhaustion of basal cells lacking $\Delta N p 63$. To determine whether the loss of basal cells occurred due to apoptosis, we stained the epithelium for the apoptotic marker, cleaved caspase 3, which showed that the increase in apoptosis observed at the 1-month timepoint was also observed at the 3-month timepoint, when up to $10 \%$ of cells were cleaved caspase 3 positive (Fig. 2o, p). The defects in the tracheal epithelium and the staining for Krt5, Ki67 and cleaved caspase 3 were quantified in at least three mice per genotype both at the 1-month (Fig. 2q, r) and 3-month timepoints (Fig. 2s, t). Taken together, our data show that $\Delta \mathrm{Np} 63$ is critical to maintain tracheal basal cells in quiescence and that loss of $\Delta N p 63$ leads to hyperproliferation followed by exhaustion of tracheal basal epithelial cells.

$\Delta \mathrm{Np63}$ is required for the regeneration and terminal differentiation of the tracheal epithelium. $\Delta \mathrm{Np} 63$ has been shown to be a crucial regulator of terminal differentiation in the skin ${ }^{11}$. To determine whether $\Delta \mathrm{Np} 63$ is similarly required for regeneration and terminal differentiation of tracheal basal cells, we tested if $\Delta N p 63$-depleted basal cells are able to differentiate into ciliated and goblet cells in vivo. To achieve this goal, we treated $\Delta N p 63^{f l f l}$; $\operatorname{Ros}^{M / M}$ mice with either adenoviral cre or adenoviral empty. After 3 days, mice were administered polidocanol (PDO) ${ }^{34}$ to deplete suprabasal tracheal epithelial cells or PBS as a control (Fig. 3a). We then analysed the regeneration of the tracheal epithelium at two timepoints after the PDO treatment, 7 days and 30 days. While the wild-type $\left(\Delta N p 63^{f l f l} ; R_{o s} a^{M / M}\right)$ basal cells were able to differentiate into a pseudostratified columnar structure of the epithelium including goblet (muc5ac positive) and ciliated (acetylated tubulin positive) cells within 7 days after the PDO challenge, the $\Delta N p 63^{\Delta / \Delta} ; \operatorname{Ros} a^{\Delta / \Delta}$ tracheal epithelial cells instead formed a disorganised epithelium of $\mathrm{Krt5}+$ cells and lacked expression of muc5ac and acetylated tubulin (Fig. 3b-i). Notably, these Krt5 + cells in the $\Delta N p 63^{\Delta / \Delta} ; \operatorname{Rosa}^{\Delta / \Delta}$ tracheal epithelium were highly proliferative with elevated apoptosis compared to the wild-type $\left(\Delta N p 63^{f l / f l} ; R o s a^{M / M}\right)$ control trachea (Fig. $\left.3 \mathrm{j}-\mathrm{m}\right)$. These data indicate that the proliferation of $\Delta N p 63^{\Delta / \Delta} ; R_{0 s a^{\Delta / \Delta}}$ tracheal epithelium at the 1-month timepoint post adenoviral cre injection (see Fig. 2f) is primarily due to basal cell proliferation and not compensatory dedifferentiation of the ciliated or goblet cells.

To determine the long-term consequences of $\Delta N p 63$ loss in tracheal epithelial cells, we examined the tracheal epithelium at 30 days after PDO administration and found that the $\Delta N p 63^{\Delta / \Delta \text {; }}$ $\operatorname{Rosa}^{\Delta / \Delta}$ mice treated with PDO had a thin, hypoplastic epithelium lacking Krt5+ cells (Fig. 3n-q). By performing immunostaining for terminal differentiation markers of tracheal epithelial cells, we found that $\Delta N p 63^{\Delta / \Delta} ; R_{o s} a^{\Delta / \Delta}$ tracheal epithelial cells were positive for acetylated tubulin similarly to the wild-type $\left(\Delta N p 63^{f l / f l} ; R o s a^{M / M}\right)$ control tracheas (Fig. $\left.3 \mathrm{r}, \mathrm{s}\right)$, but were devoid of goblet cells (Fig. 3t, u). Additionally, in contrast to the 7-day timepoint, at 30 days post-PDO administration, there were few Ki67 positive and cleaved caspase 3 positive in both genotypes (Fig. $3 \mathrm{v}-\mathrm{y}$ ). The staining for Krt5, acetylated tubulin, muc5ac, Ki67 and cleaved caspase 3 were quantified in at least three mice per genotype both at the 7-day (Fig. 3z) and 30-day timepoints (Fig. 3a'). Taken together, these data demonstrate the crucial role of $\Delta \mathrm{Np} 63$ in the maintenance of tracheal basal cells in a quiescent state and the regulation of their terminal differentiation into a goblet and ciliated cells. 
1 month
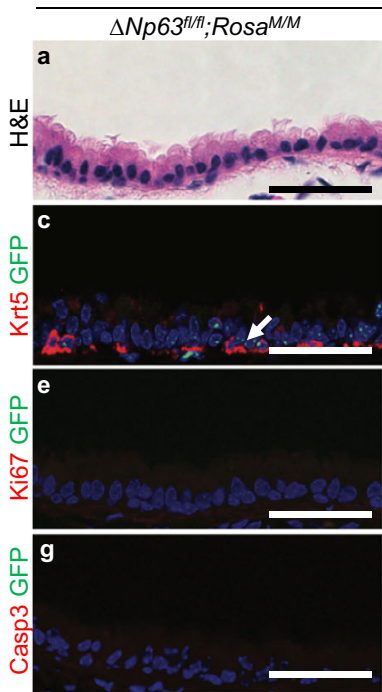

1 month quantification

q

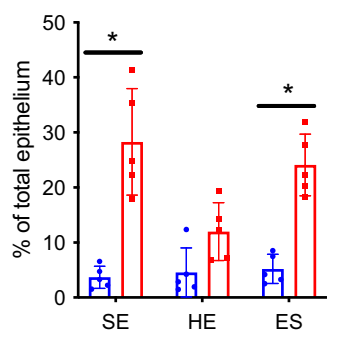

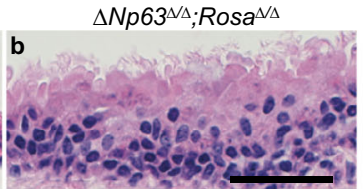
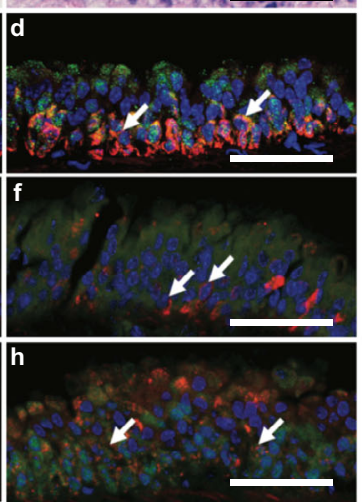

$\mathbf{r}$

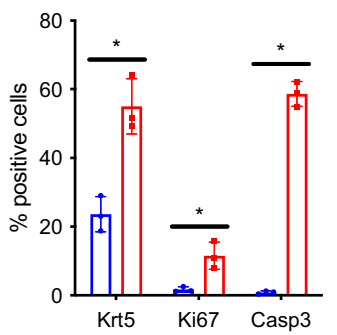

3 months
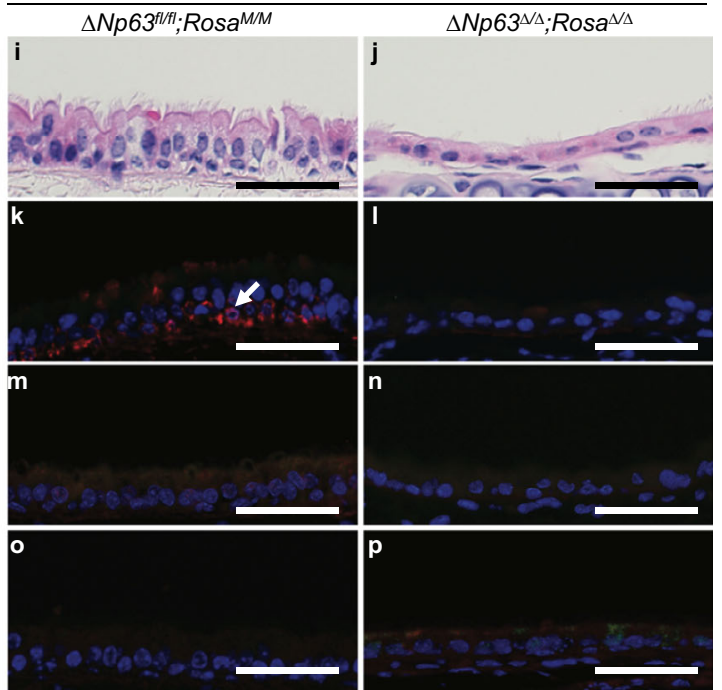

3 months quantification

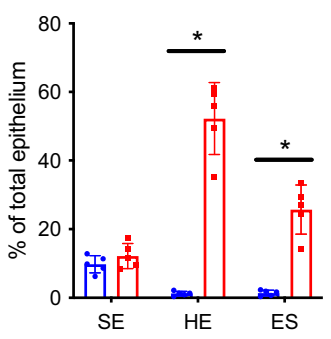

$\mathbf{t}$

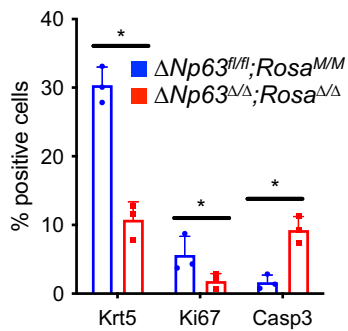

Fig. 2 In vivo $\Delta \mathbf{N p 6 3}$ ablation in the tracheal epithelium results in acute increased proliferation leading to long-term depletion of Krt5 + basal cells. a, $b$

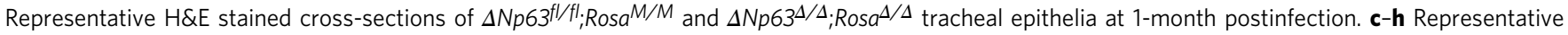
staining for Krt5/GFP (c, d), Ki67/GFP (e, f), and Casp3/GFP ( $\mathbf{g}$, h), in the same tracheal epithelia shown in $\mathbf{a}$, b. i, $\mathbf{j}$ Representative H\&E stained crosssections of $\Delta \mathrm{Np} 63^{f l / f l} ; R_{0 s a} M / M$ and $\Delta N p 63^{\Delta / \Delta} ; R_{0 s} a^{\Delta / \Delta}$ tracheal epithelia at 3 months postinfection. k-p Representative staining for Krt5/GFP (k, I), Ki67/ GFP ( $\mathbf{m}, \mathbf{n}$ ), and Casp3/GFP (o, p), in the same tracheal epithelia shown in (i, $\mathbf{j})$. All scale bars equal $100 \mu \mathrm{m}$. $\mathbf{q}$ Quantification of the stratified epithelium

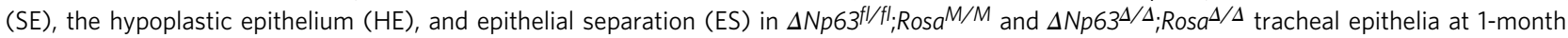

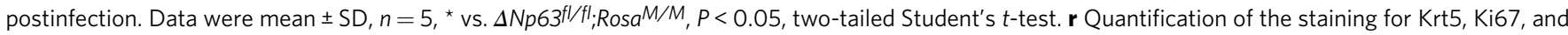

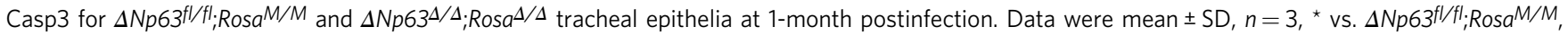
$P<0.05$, two-tailed Student's t-test. s Quantification of the stratified epithelium (SE), the hypoplastic epithelium (HE), and epithelial separation (ES) in

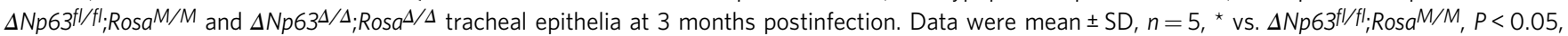
two-tailed Student's $t$-test. $\mathbf{t}$ Quantification of the staining for Krt5, Ki67, and Casp3 for $\Delta \mathrm{Np} 63^{f l / f l} ; R_{0 s a^{M} / M}$ and $\Delta \mathrm{Np} 63^{\Delta / \Delta ;}$; Rosa ${ }^{\Delta / \Delta}$ tracheal epithelia at 3 months postinfection. Data were mean \pm SD, $n=3$, * vs. $\Delta$ Np63fl/fl; Rosa ${ }^{M} / M, P<0.05$, two-tailed Student's $t$-test. Source data are provided as a Source Data file.

$\Delta \mathrm{Np63}$ is required for self-renewal of basal and distal lung stem cells. To further investigate the mechanism of $\Delta \mathrm{Np} 63$ in basal stem cell maintenance, we first isolated basal cells from $\Delta N p 63^{f l f l} ; R_{0 s} a^{M / M}$ mouse tracheas and infected them with either adenoviral cre to deplete $\Delta N p 63$ or adenoviral empty as a control. Next, we cultured them in vitro on J2-3T3 feeder cells and incubated with 5-ethynyl-2'-deoxyuridine (EdU) to determine their proliferation capacity. While at passage $1, \Delta N p 63^{\Delta / \Delta} ; \operatorname{Rosa}^{\Delta / \Delta}$ basal cell colonies replicated similarly to wild-type basal cells (Fig. 4a), at passage 2 , they became hyperproliferative and exhibited a 5- to 10 -fold increase in the $>95 \%$-positive EdU incorporation fraction compared to its wild-type counterparts (Fig. 4b). However, at passage 3, $\Delta N p 63^{\Delta / \Delta} ; \operatorname{Rosa}^{\Delta / \Delta}$ mouse tracheal cells were half as proliferative compared to the wild-type basal cells (Fig. 4c), thus mimicking the behaviour observed in vivo, where $\Delta N p 63^{\Delta / \Delta}$; $\operatorname{Ros}^{\Delta / \Delta}$ mouse tracheal cells are initially hyperproliferative and are then exhausted through apoptosis. Indeed, even in vitro, $\Delta N p 63^{\Delta / \Delta} ; \operatorname{Rosa}^{\Delta / \Delta}$ mouse tracheal cells are characterised by an increase in apoptosis compared to wild-type controls (Fig. $4 \mathrm{~d}$ ).
To investigate the effect of $\Delta N p 63$ loss on self-renewal, we cultured $\Delta N p 63^{f l f l} ; R o s a^{M / M}$ and $\Delta N p 63^{\Delta / \Delta} ; R_{0 s a^{\Delta / \Delta}}$ mouse tracheal basal cells in $3 \mathrm{D}$ and assessed their efficiency in forming tracheospheres. The cells devoid of $\Delta N p 63$ formed significantly fewer spheres over time, which were also fivefold smaller in size than the tracheospheres expressing $\Delta \mathrm{Np} 63$ (Fig. $4 \mathrm{e}-\mathrm{g}$ ). These effects were amplified by the passaging of the basal tracheospheres, indicating that $\Delta N p 63$ is required for the self-renewal of tracheal basal stem cells.

To determine whether $\Delta N p 63$ is required for tracheal basal cell differentiation in vitro, we differentiated tracheal basal cells in $3 \mathrm{D}$ matrigel culture for 20 days $^{3}$. While the wild-type tracheal basal cells formed hollow bi-layered spheres (Fig. 4h, i) comprised of an exterior layer of $\mathrm{Krt5}^{+}$and $\mathrm{NGFR}^{+}$basal cells (Fig. $4 \mathrm{j}, \mathrm{k}$ ) and an interior of ciliated and goblet cells (Fig. 4l, m), $\Delta N p 63^{\Delta / \Delta} ; R_{o s} a^{\Delta / \Delta}$ tracheal basal cells instead formed 100-fold smaller, monolayered spheres (Fig. $4 \mathrm{n}, \mathrm{o}$ ) that lacked staining for differentiation markers, including Krt5, acetylated tubulin and muc5ac (Fig. $4 \mathrm{p}-\mathrm{s}$ ). While the $\Delta N p 63^{\Delta / \Delta} ; \operatorname{Ros}^{\Delta / \Delta}$ tracheospheres lacked 


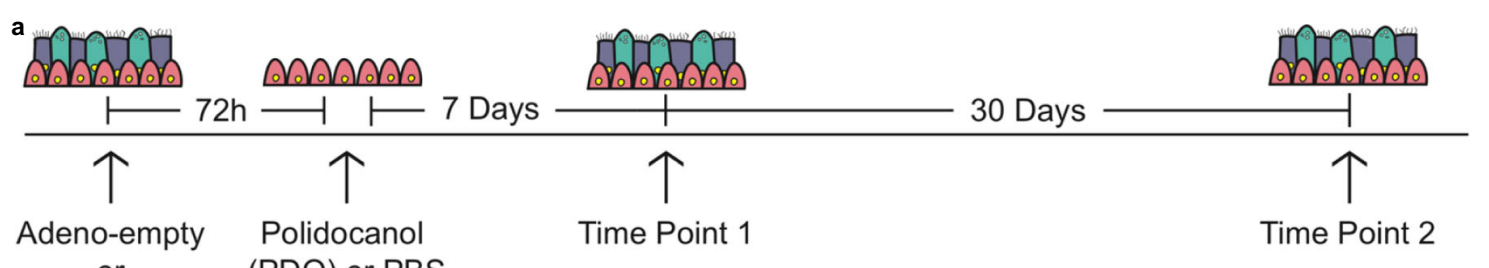

$\begin{array}{cc}\text { or } & \text { (PDO) or PBS } \\ \text { Adeno-cre } & \end{array}$

Key $\odot$ basal cell $($ Krt5+) $\quad$ o ciliated cell (Ac-tub+) $\bigcirc$ goblet cell (Muc5ac+)

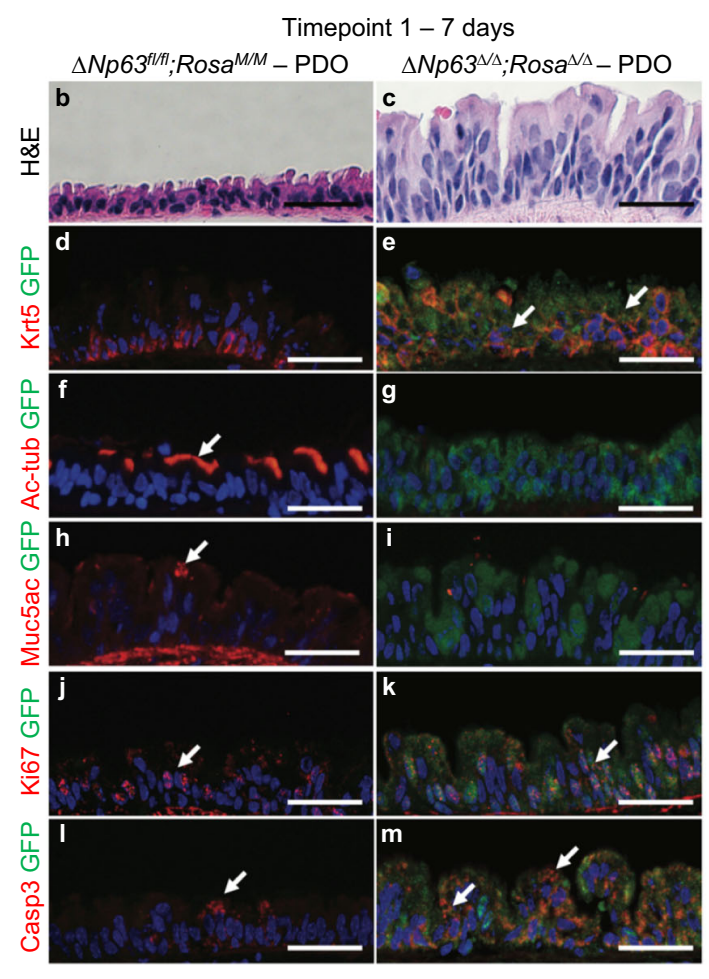

z

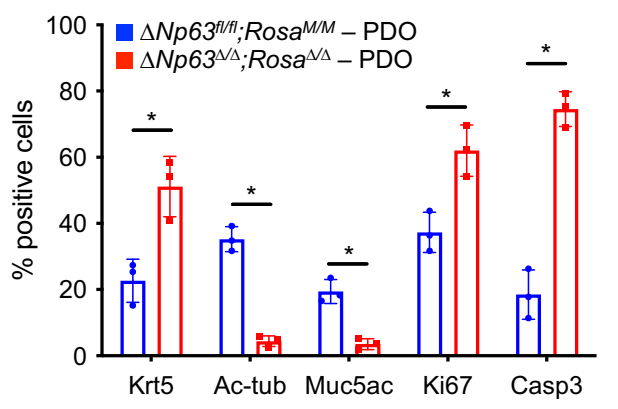

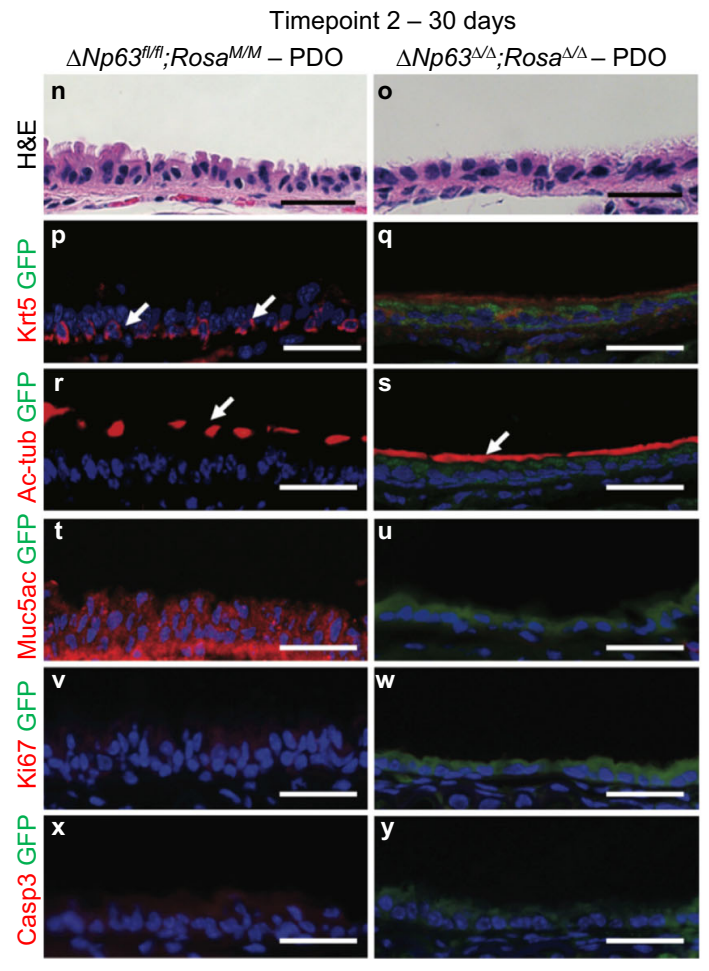

a'

Timepoint 2 - 30 days

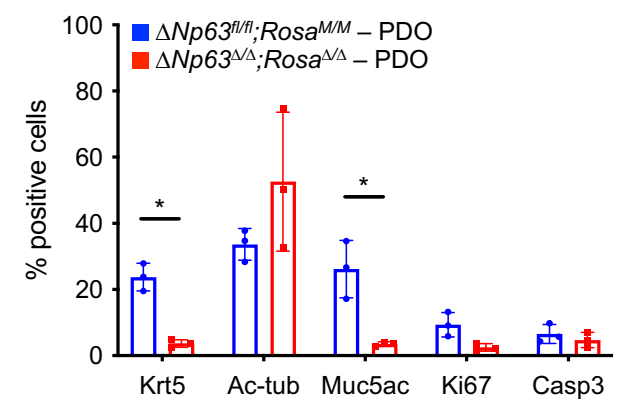

Fig. $3 \Delta$ Np63 is required for the regeneration and terminal differentiation of the tracheal epithelium. a Schematic of polidocanol tracheal injury

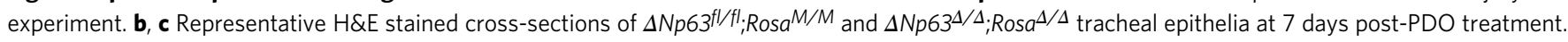
d-m Representative staining for Krt5/GFP (d, e), Ac-tub/GFP (f, $\mathbf{g})$, Muc5ac/GFP (h, i), Ki67/GFP (j, $\mathbf{k}$ ) and Casp3/GFP (I, m) in the same tracheal epithelia shown in $\mathbf{b}, \mathbf{c} . \mathbf{n}, \mathbf{o}$ Representative $\mathrm{H} \& \mathrm{E}$ stained cross-sections of $\Delta \mathrm{Np} 63^{\mathrm{fl} / \mathrm{fl} ;}$ :Rosa $\mathrm{M} / \mathrm{M}$ and $\Delta \mathrm{Np} 63^{\Delta / \Delta ;}$; Rosa ${ }^{\Delta / \Delta}$ tracheal epithelia at 30 days post-PDO treatment. p-y Representative staining for Krt5/GFP (p, q), Ac-tub/GFP (r, s), Muc5ac/GFP (t, u), Ki67/GFP (v, w) and Casp3/GFP (x, y) in the same tracheal epithelia shown in $(\mathbf{n}, \mathbf{o})$. All scale bars equal $100 \mu \mathrm{m}$. z Quantification of the staining for Krt5, Ac-tub, Muc5ac, Ki67 and Casp3 for $\Delta N p 63^{f l / f l}$;

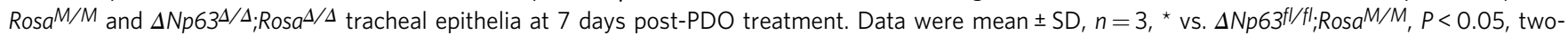

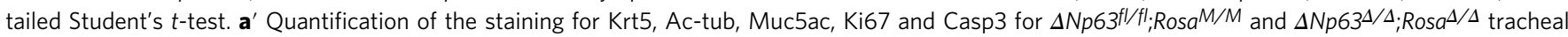
epithelia at 30 days post-PDO treatment. Data were mean $\pm S D, n=3,{ }^{\star}$ vs. $\Delta N p 63^{f / / f l} ; R o s a^{M} / M, P<0.05$, two-tailed Student's $t$-test. Source data are provided as a Source Data file. 

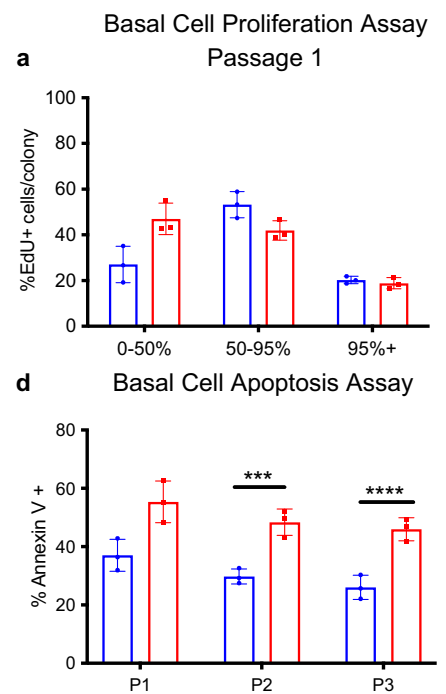

f Tracheosphere Formation Assay
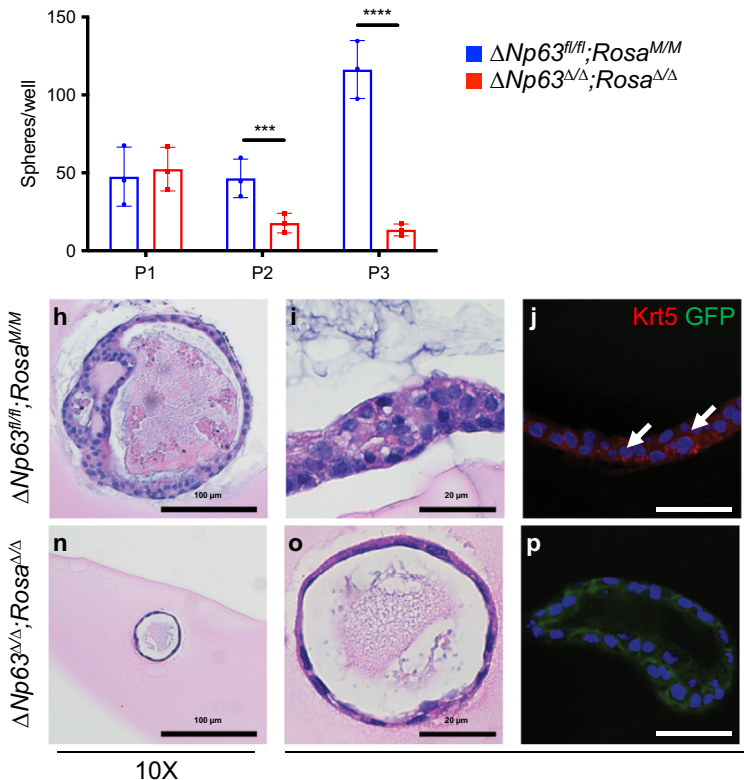

$\mathbf{t}$

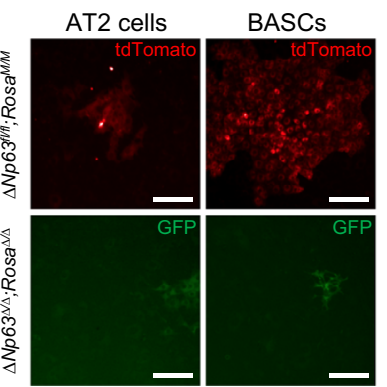

Basal Cell Proliferation Assay

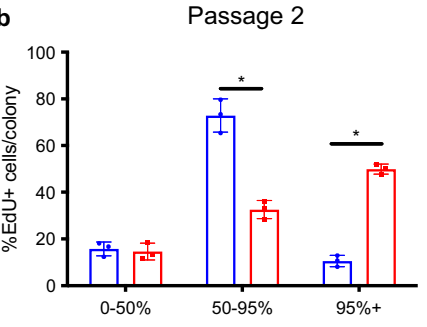

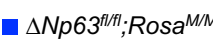

$\Delta N p 63^{\Delta \Delta} ; R^{N o s} a^{N \Delta}$

$\Delta N p 63^{f / f f l} ; R_{0} a^{M / M}$ $\Delta N p 63^{N \Delta} ; R_{0 s a}{ }^{N / \Delta}$

$$
\text { e }
$$

Basal Cell Proliferation Assay Passage 3

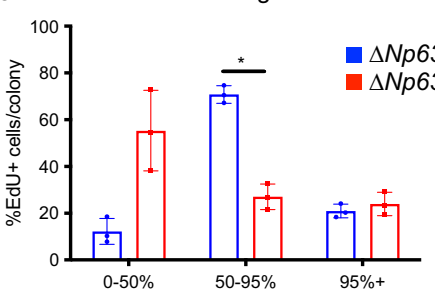

Tracheosphere Formation Assay

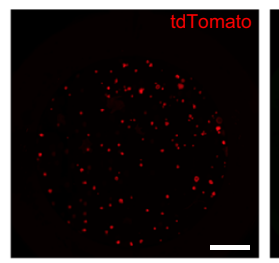

$\Delta N p 63^{f / / f l} ; R_{0 s} a^{M / M}$

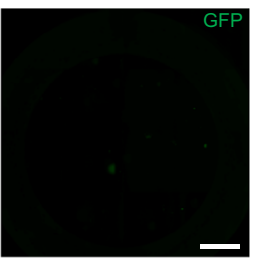

$\Delta N p 63^{\Delta / \Delta} ; \operatorname{Rosa}^{\Delta / \Delta}$

g Tracheosphere Size
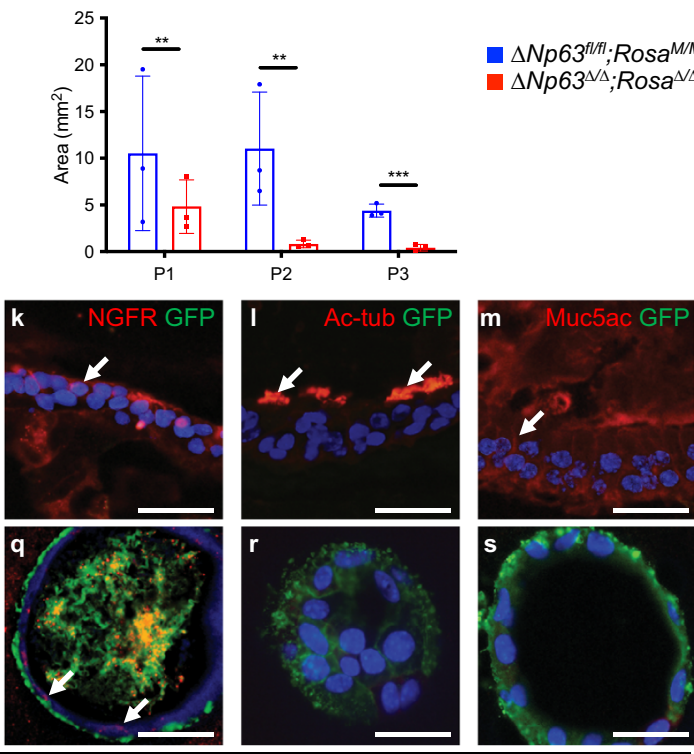

40X

u AT2 and BASC Colony Culture

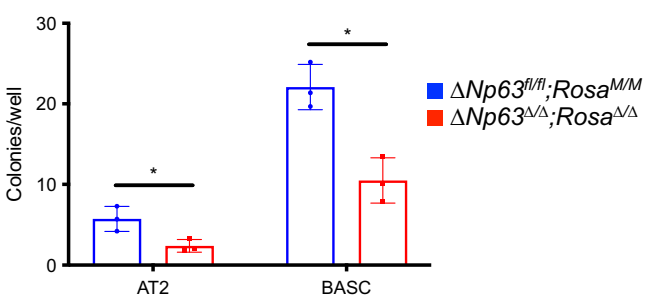

Fig. $4 \Delta$ Np63 is required for self-renewal of basal and distal lung stem cells. a-c Quantification of EdU incorporation in $\Delta N p 63^{f l} / f l ; R o s a^{M} / M$ and $\Delta N p 63^{\Delta / \Delta}$;

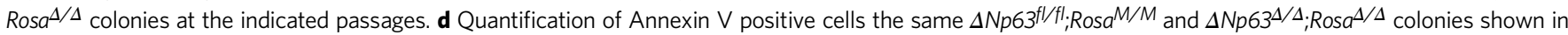

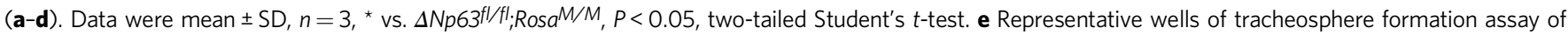

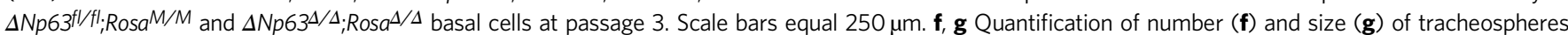

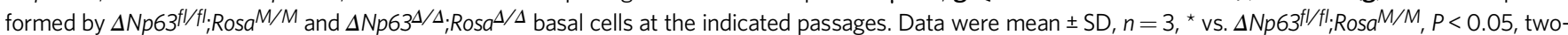

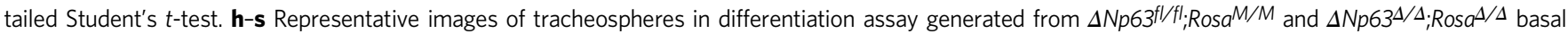
cells, showing H\&E stained cross-sections (h, i and $\mathbf{n}, \mathbf{o}$ ), and staining for Krt5/GFP (j and p), NGFR/GFP (k and q), Ac-tub/GFP (I and $\mathbf{r}$ ) and Muc5ac/GFP

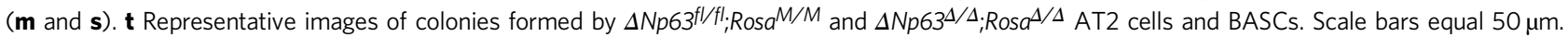
u Quantification of the same colonies as in (t). Data were mean \pm SD, $n=3$, ${ }^{\star}$ vs. $\Delta$ Np63fl/fl;:Rosa $M / M, P<0.05$, two-tailed Student's $t$-test. Source data are provided as a Source Data file. 
staining for the basal cell marker, Krt5 (Fig. 4p), an alternative marker for basal cells NGFR was expressed (Fig. 4q), demonstrating that cells lacking $\Delta N p 63$ originate from a Krt5 negative basal lineage. Taken together, our data indicate that $\Delta N p 63$ is required for self-renewal and terminal differentiation of tracheal basal cells.

Because we found that $\Delta \mathrm{Np} 63$ is required for the formation and progression of LUAD and LUSC and has a key role in maintaining tracheal basal cells, we next asked whether $\Delta \mathrm{Np} 63$ may also regulate distal lung stem cells, where the cell of origin of LUAD reside. Therefore, we isolated AT2 and BASC cells from wild-type $\left(\Delta N p 63^{f l / f l} ; R_{o s} a^{M / M}\right)$ lungs and infected them with adenoviral empty or adenoviral cre to delete $\Delta N p 63$. By performing a colony formation assay using $\Delta N p 63^{f l / f l} ; R_{0 s} a^{M / M}$ and $\Delta N p 63^{\Delta / \Delta} ; R_{0 s} a^{\Delta / \Delta}$ AT2 and BASC cells, we found that the loss of $\triangle N p 63$ resulted in a twofold decrease in colony formation of AT2 cells and BASCs (Fig. 4t, u). Taken together, our results demonstrate that $\triangle N p 63$ is essential for the proper maintenance of the different lung stem cell populations, including basal cell, AT2, and BASC cells, and for the terminal differentiation of tracheal basal cells into a goblet and ciliated cells.

\section{$\Delta N p 63$ regulates the enhancer landscape of cell identity genes} in basal cells of the trachea. Many transcription factors required for the self-renewal, maintenance and identity of progenitor and stem cells have been shown to regulate the enhancer landscape of genes that control these processes ${ }^{35-37}$. These enhancers, marked by acetylated $\mathrm{H} 3 \mathrm{~K} 27$ (H3K27ac) in the presence of monomethyl $\mathrm{H} 3 \mathrm{~K} 4$ (H3K4me1), highly upregulate gene expression and are linked to the regulation of cell identity, stem cell pluripotency and cancer states ${ }^{37-39}$. To verify whether $\Delta \mathrm{Np} 63$ regulates selfrenewal and terminal differentiation of tracheal basal cells by controlling the enhancer landscape, we isolated primary tracheal basal cells from $\Delta N p 63^{f l / f l} ; \operatorname{Ros} a^{M / M}$ and $\Delta N p 63^{\Delta / \Delta} ; R o s a^{\Delta / \Delta}$ and performed RNA-seq and chromatin immunoprecipitation (ChIP)-seq analysis (Fig. 5a). Pathway analysis was performed using the differentially expressed genes identified from the RNAseq data. Most of the pathways affected in $\triangle \mathrm{Np} 63$ deficient tracheal basal cells are involved in proliferation, stem cell maintenance and adhesion (Fig. 5b and Supplementary dataset 1). To identify direct transcriptional targets of $\Delta \mathrm{Np} 63$ in the tracheal basal cells, we performed low-cell number ChIP-seq analysis using an antibody directed against $\Delta \mathrm{Np} 63$, which allowed us to generate a $\Delta \mathrm{Np} 63$ binding motif similar to the previously published p63 motif ${ }^{40}$ (Fig. 5c). To identify enhancers regulated by $\Delta \mathrm{Np63}$, we also performed ChIP-seq for RNA polymerase 2 (Pol2) and H3K27ac. Importantly, we found that lack of $\Delta N p 63$ affected genome-wide $\mathrm{H} 3 \mathrm{~K} 27 \mathrm{ac}$ and $\mathrm{Pol} 2$ occupancy, especially at the most active enhancers (Fig. 5d, e, Supplementary Fig. 2a and Supplementary dataset 2). Comparison of the H3K27ac ChIP-seq signal revealed that the top 500 enhancers were significantly decreased upon deletion of $\triangle N p 63$, with a higher H3K27ac signal observed at regions containing the $\Delta$ Np63 motif (Fig. 5d). Among the genes associated with the top 2000 enhancers in basal cells, there are genes involved in epithelialization and cell junction maintenance (Fig. 5e, $\mathrm{f}$ and Supplementary dataset 2). In cells expressing $\triangle N p 63, \Delta$ Np63-regulated genes such as $K r t 5$ and $B C L 9 L$ are associated with a robust $\mathrm{H} 3 \mathrm{~K} 27 \mathrm{ac}$ signal, including in enhancer regions reported in the Fantom 5 enhancers ${ }^{41}$ and the Mouse Encode lung enhancers ${ }^{42}$ catalogues (Fig. $5 \mathrm{~g}$, $\mathrm{h}$ and Supplementary dataset 3 ). These data indicate that $\Delta \mathrm{Np} 63$ regulates the expression of lung basal cell identity genes by binding to and affecting the acetylation status of the corresponding enhancers.
$\Delta$ Np63 regulates self-renewal by controlling the enhancer landscape of cell identity genes in distal lung stem cells. Given the crucial role of $\Delta \mathrm{Np} 63$ in the regulation of the enhancer landscape of cell identity genes of tracheal basal cells, we asked whether $\Delta \mathrm{Np} 63$ is playing a similar role in lung distal progenitor cells. To address this, we performed low-cell number ChIP-seq for $\mathrm{H} 3 \mathrm{~K} 27 \mathrm{ac}$, Pol2 and $\Delta \mathrm{Np} 63$, in AT2 cells isolated from $\Delta N p 63^{f l / f l} ; R_{0 s} a^{M / M}$ lungs and infected with adenoviral empty or adenoviral cre to delete $\Delta N p 63$ (Fig. 6a). Similar to what was found in basal cells (see Fig. 5c), also in AT2 cells the $\Delta \mathrm{Np} 63$ binding motif was comparable to the previously published p63 motif $^{40}$ (Fig. 6b). While we did not observe a genome-wide reduction in the $\mathrm{H} 3 \mathrm{~K} 27 \mathrm{ac}$ occupancy (Supplementary Fig. $2 \mathrm{~b}$ and Supplementary dataset 3), ranking of genes based on the H3K27ac signal identified genes associated with the top 2000 enhancers in AT2 cells that had decreased expression after the loss of $\triangle N p 63$, including BCL9L and ETV5 (Fig. 6c, d and Supplementary dataset 4 ). Interestingly, $B C L 9 L$ was controlled by $\triangle$ Np63 also in the tracheal basal cells (see Fig. 5f), while ETV5 is an essential gene for maintenance of the AT2 cell population ${ }^{43}$. Importantly, the ChIP-seq peaks revealed that the recruitment of $\triangle \mathrm{Np} 63$ is associated with regions of increased H3K27ac signal in both the ETV5 and BCL9L loci (Fig. 6d, e). Taken together, our findings demonstrate that $\triangle \mathrm{Np} 63$ also regulates the enhancer landscape of cell identity genes in the distal lung stem cell population and that this regulation may be important for its oncogenic role.

$\Delta$ Np63 regulates a common landscape of enhancer-associated genes in tracheal basal cells, AT2 cells and LUAD. Given that $\Delta$ Np63 regulates the enhancer landscape in tracheal basal cells and AT2 cells, we asked whether there were any common enhancers regulated in both cell types that may shed light on its function as a common regulator of stem cell identity and oncogenesis. Cross comparison of the datasets derived from tracheal basal cells and AT2 cells indicated that the top 2000 enhancer signatures from both cell types shared 5326 common genes (Fig. 7a and Supplementary dataset 5) that may mediate the common function of $\Delta \mathrm{Np} 63$ in tumour maintenance. To enable translation from our mouse data into human samples, we performed a mouse-human cross-species analysis of the identified genes using $\mathrm{H} 3 \mathrm{~K} 27 \mathrm{ac}$ ChIP-seq data from human LUAD and LUSC. In each of these datasets, we ranked the top 2000 enhancers and queried genes located near the enhancer regions that also contained an $\Delta \mathrm{Np} 63$ binding motif. Genes that met these criteria were substantiated with the $\mathrm{LUAD}^{44}$ and $\mathrm{LUSC}^{19}$ TCGA databases for alterations in cancer. Using these criteria, we found Krt5 (a basal cell-specific gene), ETV5 (an AT2-specific gene) and BCL9L (one of the genes in common between the basal cell and the AT2 signatures) as significant in both mouse and human datasets.

To determine whether $\Delta \mathrm{Np} 63$ directly regulates the enhancers of cell identity genes, we employed a CRISPR-dCas9 (the nuclease-null deactivated Cas9) based system ${ }^{45}$. Specifically, we transfected $29 \overline{3}$ T cells with vectors expressing dCas9-conjugated $\triangle \mathrm{Np} 63$ that were targeted to enhancer regions via short guide RNAs (sgRNAs) corresponding to the $\Delta$ Np63 motif (Fig. $7 \mathrm{~b}$ ). dCas9-p300 was also included, since the histone acetyltransferase p300 also marks the most active enhancer regions ${ }^{46}$. Four sgRNAs were designed for targeting an $\triangle \mathrm{Np} 63$ motif within the identified enhancer regions of the Krt5, ETV5 and BCL9L loci. While there was no significant effect with dCas9-p300 alone at identified enhancers, dCas9- $\triangle \mathrm{Np} 63$ resulted in robust gene expression of ETV5, BCL9L and KRT5 by 3, 7 and 13 folds 
a
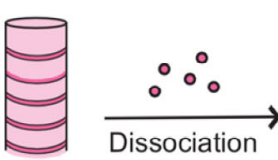

$\Delta \mathrm{Np}^{\mathrm{nt} / \mathrm{fl}} ;$ Rosam$^{\mathrm{M} / \mathrm{M}}$

pooled tracheas

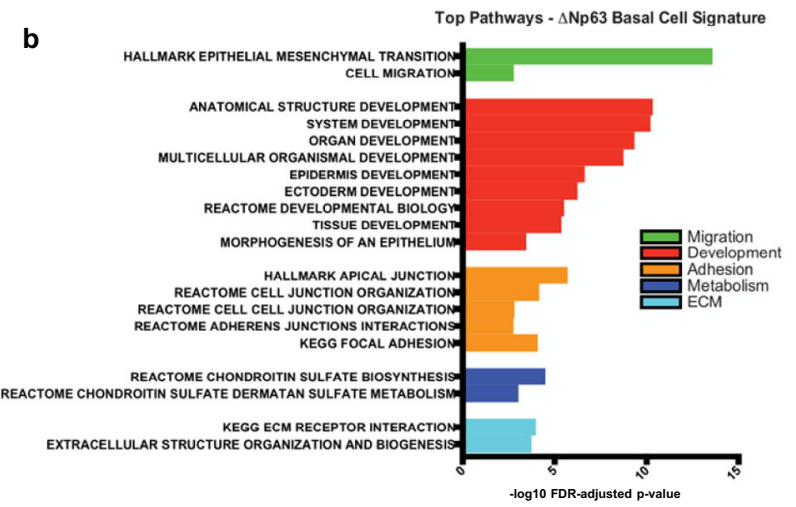

d

Basal Cell H3K27ac at top 500 enhancers

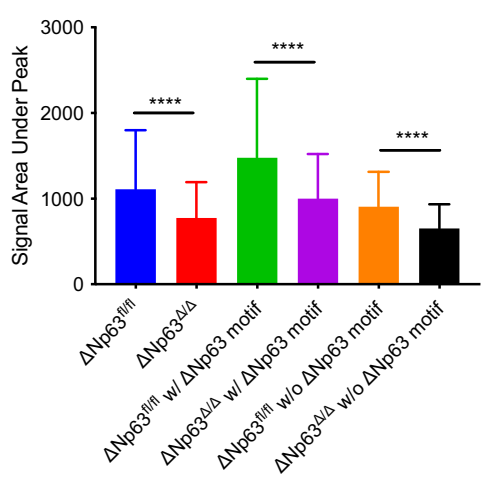

g

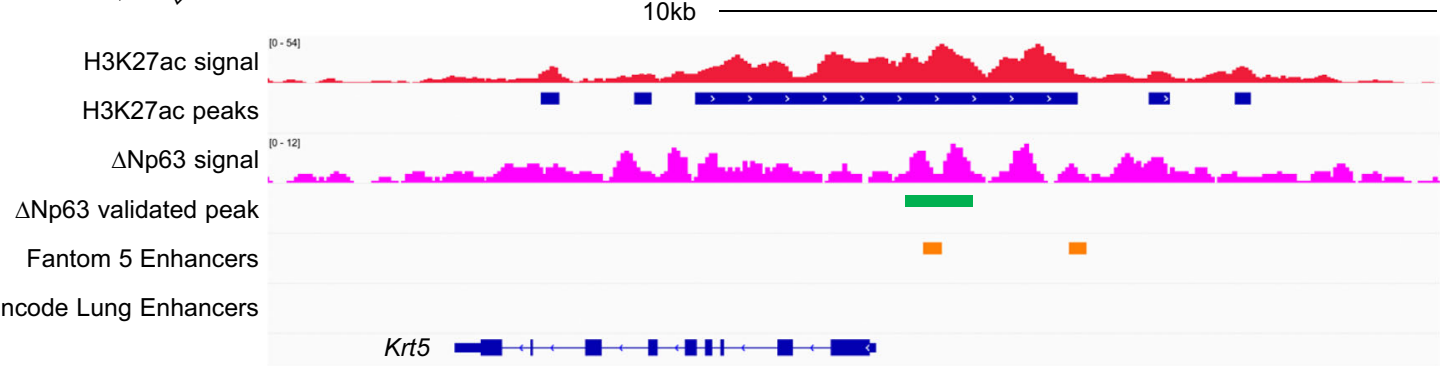

h

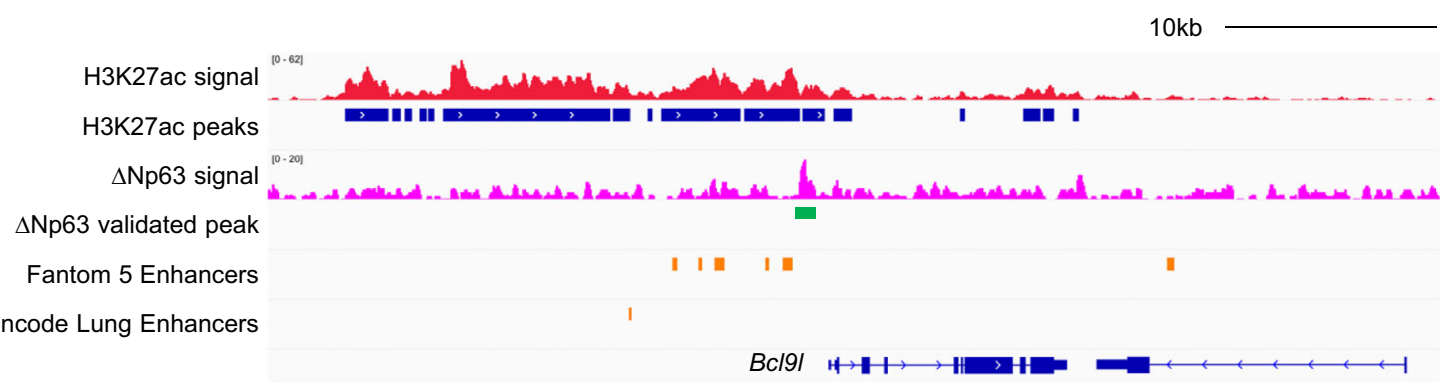

respectively (Fig. 7c-e). Moreover, the combination of dCas9$\Delta \mathrm{Np} 63$ and dCas9-p300 recruitment further increased gene expression significantly over dCas $9-\Delta \mathrm{Np} 63$ alone, demonstrating that both $\Delta \mathrm{Np} 63$ and p300 are recruited to these enhancer regions and are necessary to induce the expression of the corresponding gene (Fig. $7 \mathrm{c}-\mathrm{e}$ ).
Adeno-empty

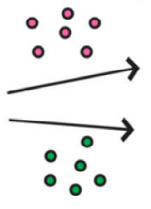

Adeno-cre

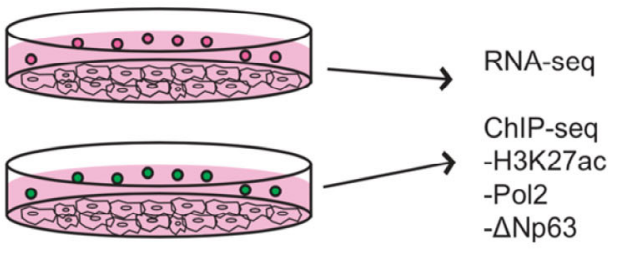

c

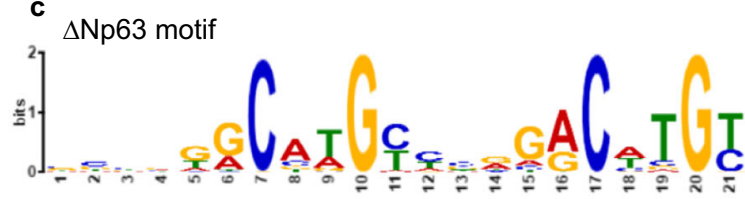

p63 motif

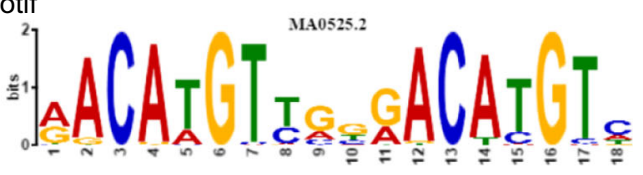

f Basal Cell gene expression

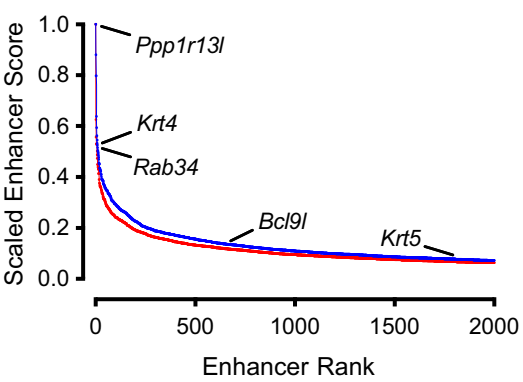

Mouse Encode Lung Enhancers

BClgl H, $\rightarrow$ H $>$ H

To further evaluate whether $\Delta \mathrm{Np} 63$ and p300 localise at $\Delta \mathrm{Np63}$ motifs located in enhancer regions, we performed ChIP in the H520 LUSC cell line, which expresses high levels of $\Delta$ Np63. When $\Delta \mathrm{Np} 63$ was targeted via shRNA, the occupancy levels of H3K27ac were significantly decreased compared to the control at the enhancers of ETV5 (4.5-fold), BCL9L (3.8-fold) and KRT5 
Fig. $5 \Delta$ Np63 regulates the enhancer landscape of cell identity genes in basal cells of the trachea. a Schematic of isolation of $\Delta N p 63^{f l / f l} ; R o s a^{M / M}$ and

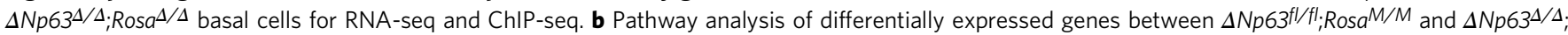
Rosa $a^{\Delta / \Delta}$ basal cells. c $\Delta$ Np63 motif derived from basal cell $\Delta$ Np63 ChIP-seq compared to published p63 motif. $\mathbf{d}$ H3K27ac ChIP-seq signal area under peak

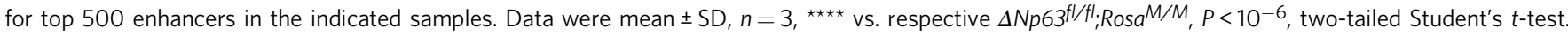
e Ranking of top 2000 enhancers based on H3K27ac ChIP-seq in $\Delta \mathrm{Np} 63^{f / / f l} ; R_{0 s} a^{M / M}$ (blue) and $\Delta N p 63^{\Delta / \Delta ; R o s a}{ }^{\Delta / \Delta}$ (red) basal cells. f qRT-PCR of the

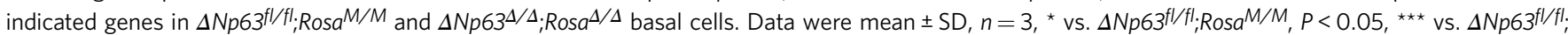
Rosa M/M, $P<0.0001$, two-tailed Student's t-test. $\mathbf{g}$, h ChIP-seq profiles of $\Delta \mathrm{Np} 63^{f l / f l}$; Rosa $\mathrm{M} / \mathrm{M}$ basal cells for the indicated tracks in the Krt5 (g) and Bcl9l (h) loci. Source data are provided as a Source Data file.

a

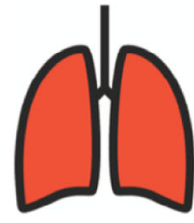

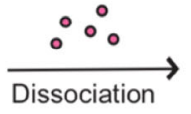

b

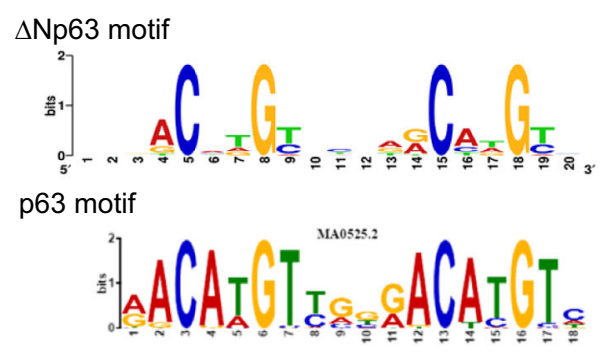

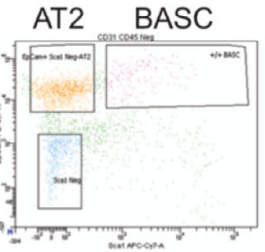

c

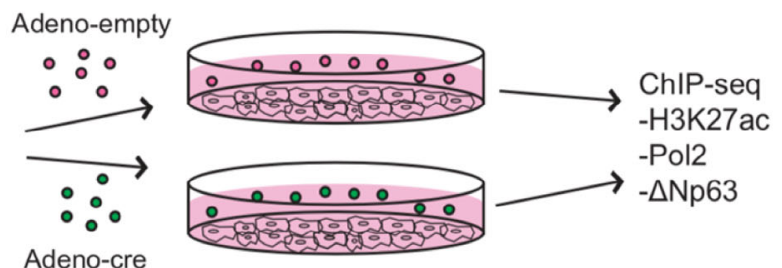

d

AT2 gene expression

$\Delta N p 63^{f / / f l} ; R_{0 s a}{ }^{M / M} \square \Delta N p 63^{\Delta / \Delta} ; R_{0 s a}{ }^{\Delta / \Delta}$

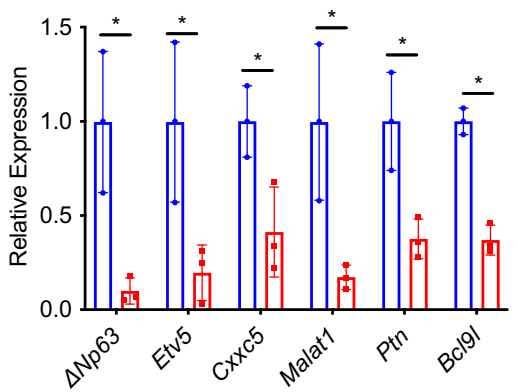

e
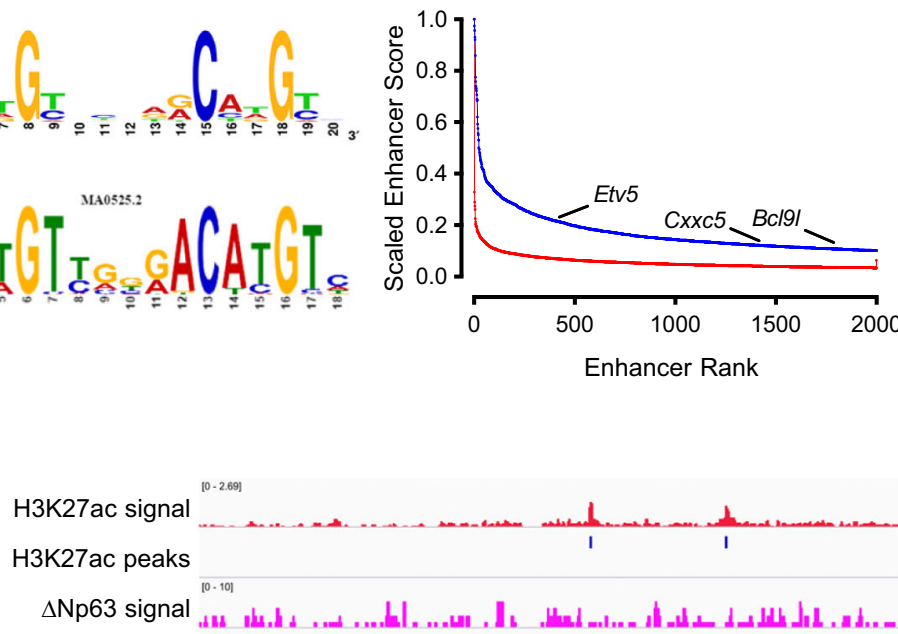

Allth

$\Delta$ Np63 validated peak

Fantom 5 Enhancers

Mouse Encode Lung Enhancers

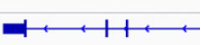

f

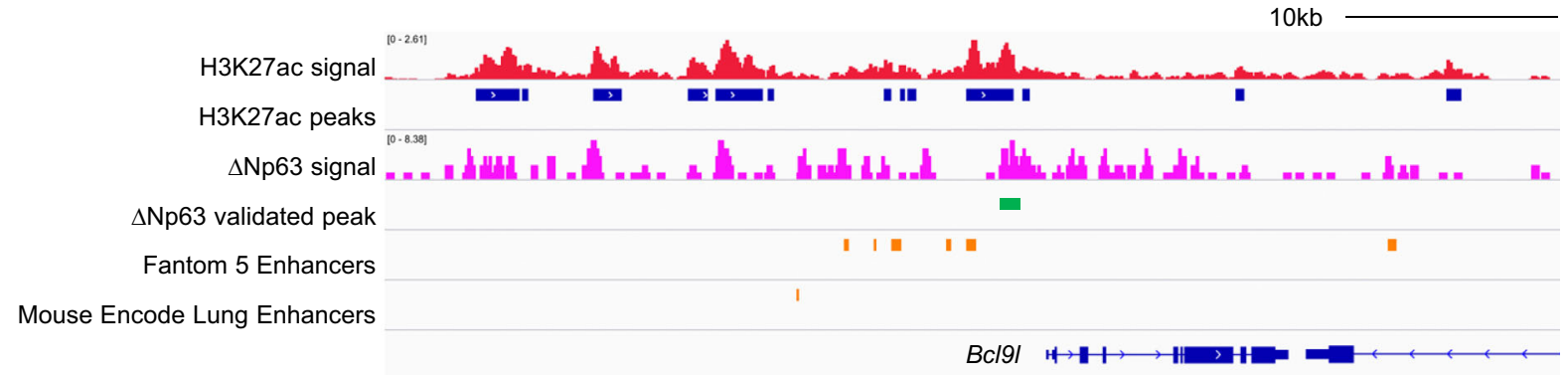

Fig. $6 \Delta$ Np63 regulates self-renewal by controlling the enhancer landscape of cell identity genes in distal lung stem cells. a Schematic of isolation of

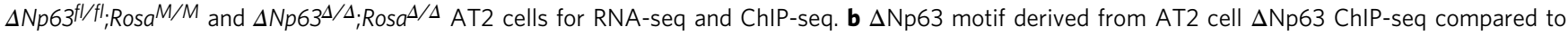

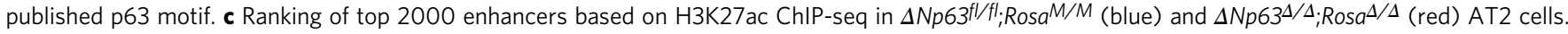
d qRT-PCR of the indicated genes in $\Delta N p 63^{f / / f l} ; R o s a^{M} / \mathrm{M}$ and $\Delta N p 63^{\Delta / \Delta} ; R o s a^{\Delta / \Delta}$ AT2 cells. Data were mean $\pm S D, n=3,{ }^{*} v s . \Delta N p 63^{f l / f l}, P<0.05$, two-tailed Student's t-test. e, $\mathbf{f}$ ChIP-seq profiles of $\Delta \mathrm{Np} 63^{f / / f l}$; RosaM/M AT2 cells for the indicated tracks in the Etv5 (e) and Bc/9l (f) loci. Source data are provided as a Source Data file. 
a

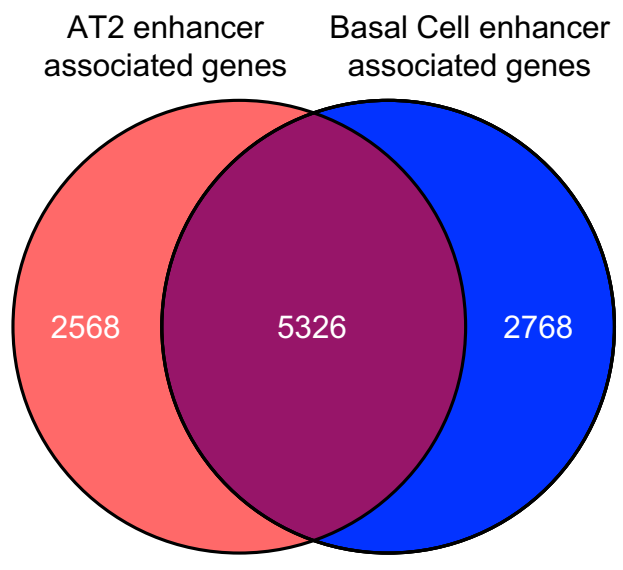

b

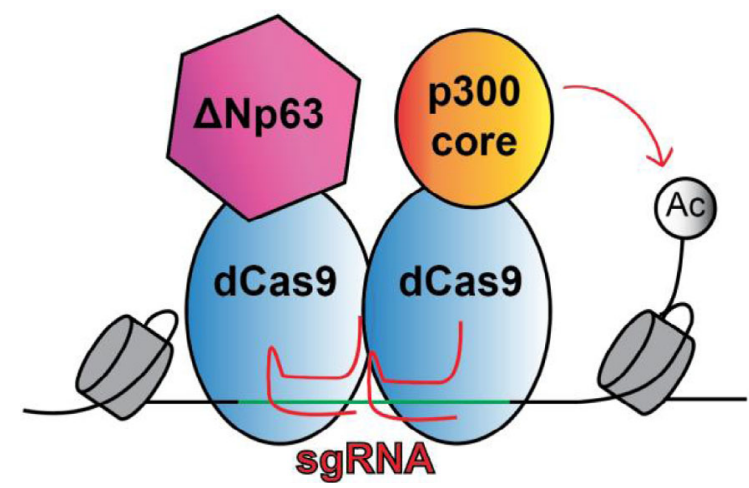

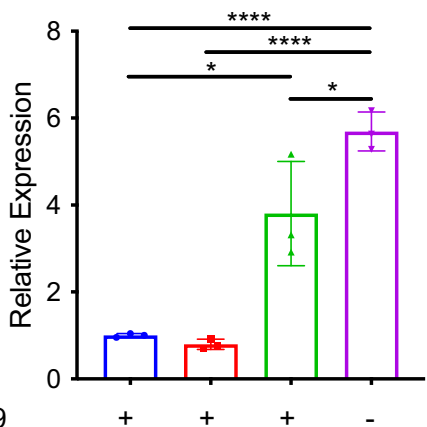

dCas9

$\mathrm{dCas} 9 \mathrm{p} 300$ core $-\quad+\quad+$

dCas9 ${ }^{\triangle N p 63}$

f

$E T V 5-\Delta N p 63$ binding motif

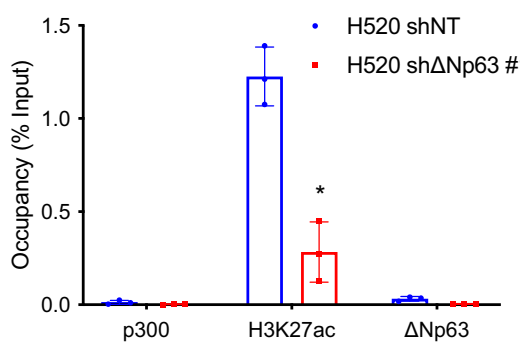

d

$B C L 9 L-\triangle \mathrm{Np63}$ binding motif

e

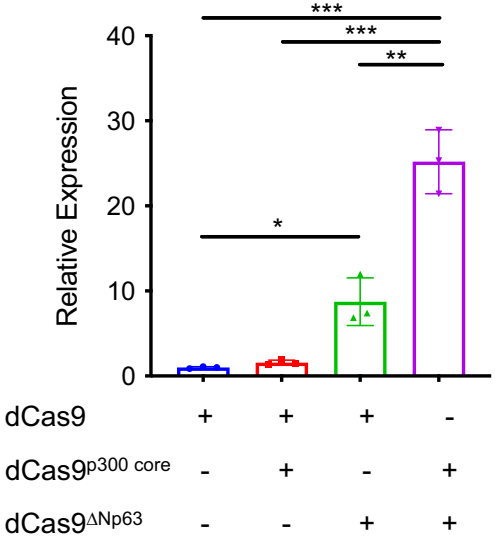

g

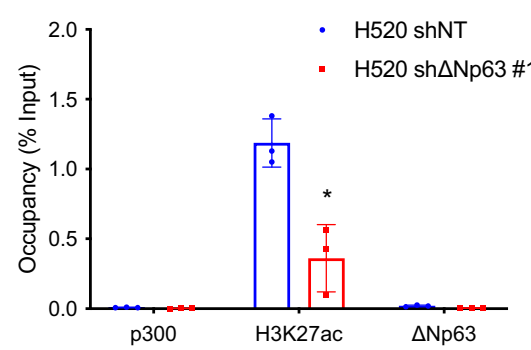

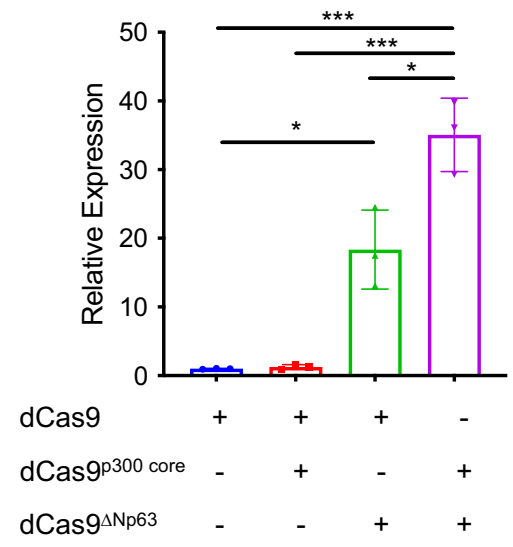

h

KRT5 $-\triangle$ Np63 binding motif

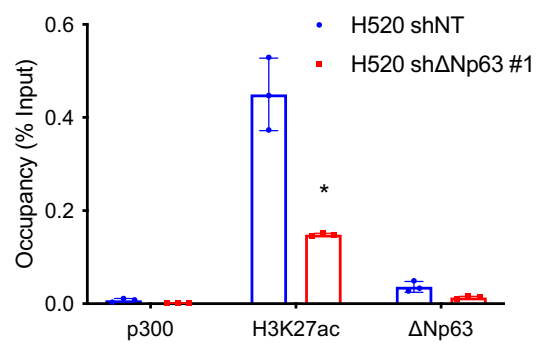

Fig. $7 \Delta$ Np63 regulates a common set of enhancer-associated genes in tracheal basal cells, AT2 cells and LUAD. a Venn diagram overlaying the genes associated with the top 2000 enhancers in AT2 and basal cells. b Schematic of dCas9-p300 and dCas9- $\Delta$ Np63 sgRNA experiment. c-e Relative expression levels of ETV5 (c), BCL9L (d) and KRT5 (e) in 293 T cells over-expressing the indicated constructs. Data were mean \pm SD, $n=3,{ }^{\star} P<0.05$, ${ }^{\star \star} P<0.01,{ }^{\star \star \star} P<0.001,{ }^{\star \star \star \star} P<0.0001$, two-tailed Student's $t$-test. $\mathbf{f}-\mathbf{h}$ qRT-PCR of ChIP assays for $\mathrm{p} 300, \mathrm{H} 3 \mathrm{~K} 27 \mathrm{ac}$ and $\Delta$ Np63 at the indicated sites. Data were mean $\pm S D, n=3$, * vs. ShNT, $P<0.05$, two-tailed Student's $t$-test. Source data are provided as a Source Data file.

(2.8-fold) (Fig. 7f-h). Taken together, these results indicate that $\Delta$ Np63 cooperates with $\mathrm{p} 300$ to regulate the enhancer landscape of lung cell identity genes involved in LUAD and LUSC.

$B C L 9 L$ mediates the oncogenic activities of $\triangle \mathrm{Np} 63$ and is critical for the maintenance of LUSC and LUAD. The fact that $\triangle \mathrm{Np63}$ regulates common enhancer-associated genes in LUSC and LUAD suggests a common mechanism for its function as an oncogene. To understand whether these genes are essential for the oncogenic functions of $\triangle \mathrm{Np} 63$ in LUSC and LUAD, we used shRNA to knockdown expression of the basal cell-specific gene KRT5, the AT2-specific gene ETV5 and BCL9L, which is one of the genes in common between the basal cell and the AT2 signatures, in the H520 LUSC cell line and the H358 LUAD cell line. Notably, in an in vitro soft agar assay, we found that knocking down the common gene, BCL9L, or the basal cell gene, $K r t 5$, significantly reduced soft agar colony formation in the H520 
a

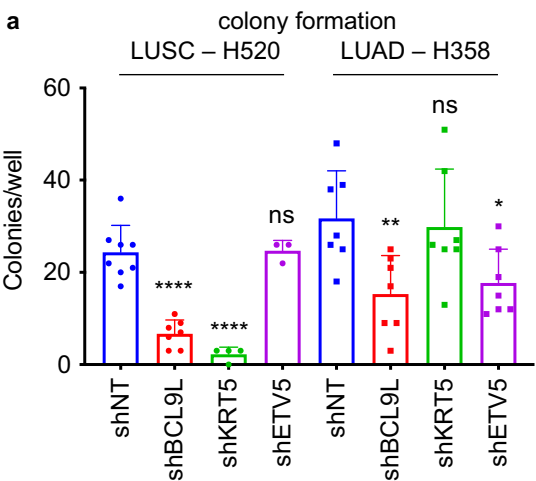

b

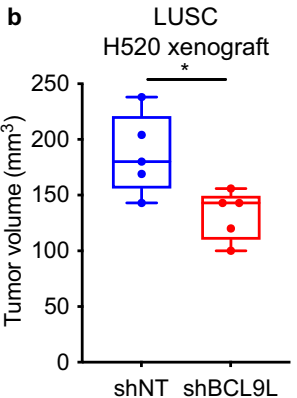

LUAD

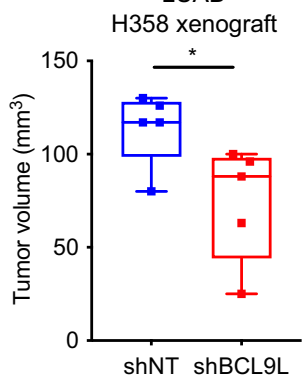

c
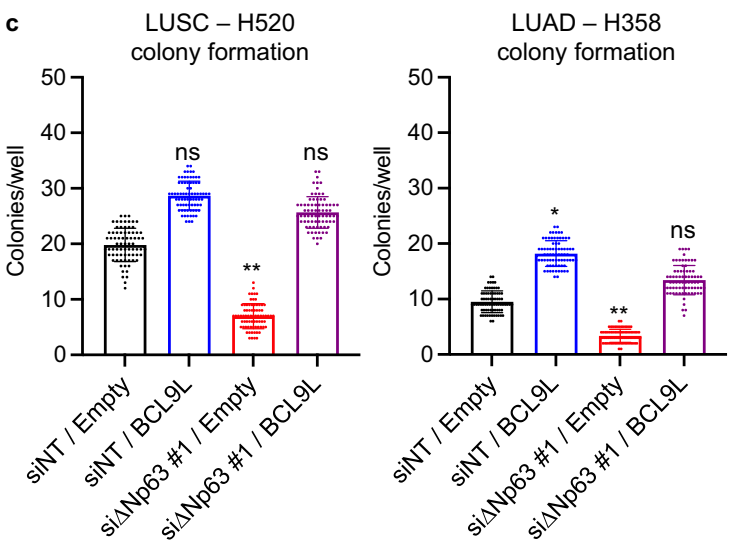

d

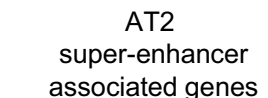

AT2 associated genes

Kras $^{\text {G12D/+ LUAD }}$

super-enhancer associated genes

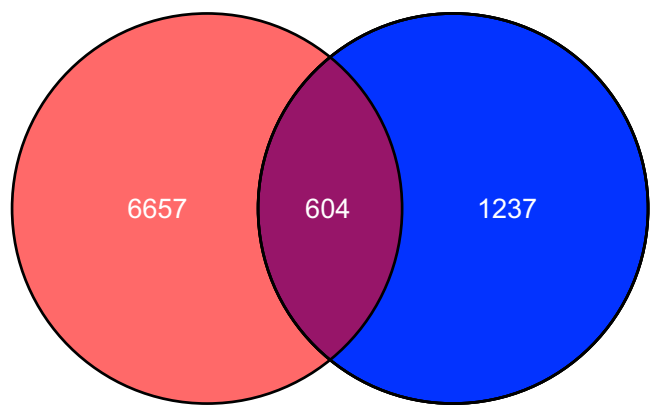

$\mathbf{f}$

Survival - TCGA LUAD cohort
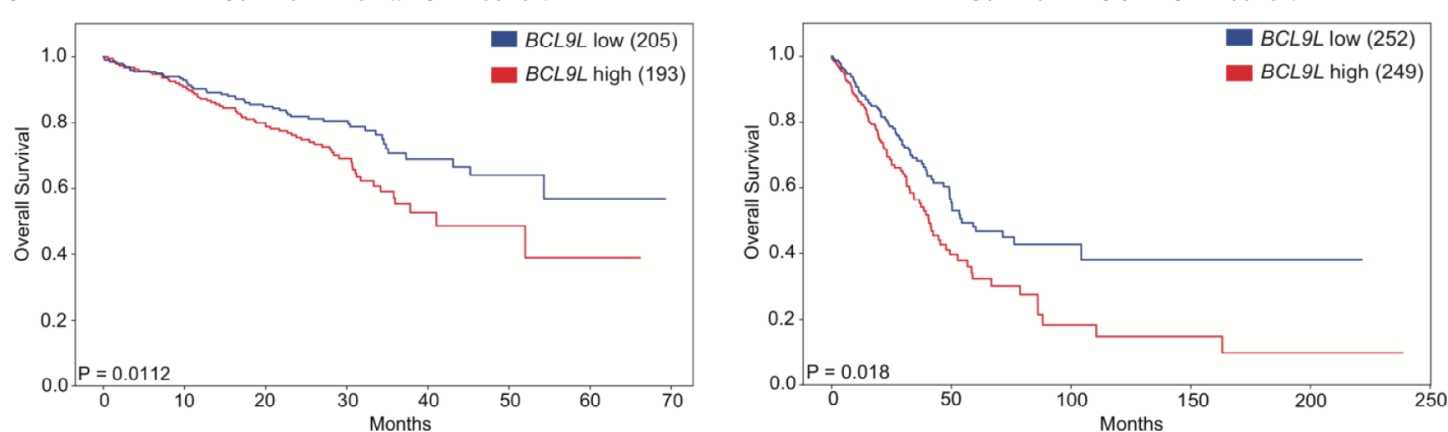

Fig. 8 BCL9L mediates the oncogenic activities of $\triangle N$ p63 and is critical for maintenance of LUSC and LUAD. a Quantification of colony formation efficiency in soft agar of H520 LUSC cells and H358 LUAD cells infected with the indicated shRNAs. Data were mean \pm SD, $n=3,{ }^{\star} P<0.05,{ }^{\star \star} P<0.01$, ${ }^{\star \star \star \star} P<0.0001$, vs. respective shNT, two-tailed Student's $t$-test. $\mathbf{b}$ Tumour volume quantification of xenograft tumours derived from the indicated cell lines. Boxplots represent the individual data points, median and whiskers (min to max method) are shown, $n=5,{ }^{\star}$ vs. respective shNT, $P<0.05$, two-tailed Student's $t$-test. c Quantification of colony formation efficiency in soft agar of H520 LUSC cells and H358 LUAD cells transfected with the indicated constructs. Boxplots represent the individual data points, median and whiskers (min to max method) are shown, $n=3,{ }^{\star} P<0.05,{ }^{\star \star} P<0.01$, vs. respective siNT/empty, two-tailed Student's $t$-test. d Venn diagram overlaying genes associated with the top 2000 enhancers in AT2 cells and LUAD derived from Kras $G 12 \mathrm{D} /+$ mice. e, $\mathbf{f}$ Kaplan-Meier curves of overall lung adenocarcinoma survival data based on the levels of BCL9L in the indicated cohorts. Source data are provided as a Source Data file.

LUSC cell line, whereas knockdown of the AT2-specific gene, $E T V, 5$ did not produce a significant effect (Fig. 8a), thus indicating that regulation of $B C L 9 L$ and KRT5 by $\triangle \mathrm{Np} 63$ is critical for maintenance of LUSC. Conversely, knocking down the common gene, BCL9L, or AT2-specific gene, ETV5, significantly reduced soft agar colony formation in the H358 LUAD cell line, whereas knockdown of the basal cell-specific gene, KRT5, did not produce a significant effect (Fig. 8a). These data indicate that $B C L 9 L$ and ETV5 are $\triangle \mathrm{Np} 63$ targets critical for the maintenance of LUAD. To further demonstrate the importance of $B C L 9 L$ in LUSC and LUAD in vivo, we injected the H520 LUSC cells or the H358 LUAD cells expressing shBCL9L or shNT used as a negative control into the flank of nude mice. Importantly, we found that knockdown of BCL9L impaired tumour formation in both cell types (Fig. 8b), further indicating that BCL9L is an $\triangle \mathrm{Np63-regulated} \mathrm{oncogene.} \mathrm{To} \mathrm{test} \mathrm{the} \mathrm{role} \mathrm{of} \mathrm{BCL9L} \mathrm{in} \mathrm{the} \mathrm{pro-}$ oncogenic activities of $\Delta \mathrm{Np} 63$, we performed soft agar assays in two LUSC cell lines (H520 and H2170) and three LUAD cell lines (H358, H1944 and H2009). In all the tested cell lines, downregulation of $\triangle N p 63$ decreased the expression levels of $B C L 9 L$ (Supplementary Fig. $3 \mathrm{a}-\mathrm{f}$ ) and significantly reduced the in vitro colony formation (Fig. 8c and Supplementary Fig. 4a-e). Notably, the overexpression of myc-tag BCL9L was able to rescue the anchorage-independent ability in all the five cell lines (Fig. $8 \mathrm{c}$ and 
Supplementary Fig. 4a-e), indicating that BCL9L is an important mediator of the oncogenic effects of $\triangle \mathrm{Np} 63$ in both LUAD and LUSC. The oncogenic role of BCL9L in lung cancers is also supported by the comparison of $\triangle \mathrm{Np} 63$-regulated enhancers associated genes in AT2 cells and in LUAD derived from tumours of $K r a s^{G 12 D /+}$ mice, showing that BCL9L is included in the common gene set (Fig. 8d and Supplementary dataset 6). Finally, to evaluate the relevance of $B C L 9 L$ as an oncogene in human lung cancers, we analysed its prognostic value in two independent cohorts of LUAD and LUSC. Importantly, in both LUAD cohorts, high levels of $B C L 9 L$ correlated with a poorer prognosis compared to low levels of BCL9L (Fig. 8d, e). In both LUSC cohorts, a similar trend was observed, even though it was not significant. Taken together, this further indicates that the regulation of the $B C L 9 L$ enhancer landscape by $\triangle \mathrm{Np} 63$ is critical for its function as an oncogene in both LUSC and LUAD and for the first time provides a common mechanism for the $\Delta \mathrm{Np} 63$ functions as an oncogene in NSCLC.

\section{Discussion}

Recent work has identified epigenetic mechanisms of gene regulation as key modulators of cancer progression ${ }^{47}$. In particular, enhancers are considered key regulators of spatiotemporal gene expression, with the most active enhancers being frequently associated with genes that designate cell identity ${ }^{38}$. Interest has grown in understanding the regulation of genes associated with the most active enhancers, since these genes are the most highly expressed in the cell and are thought to play a key role in the biology of cancer. Here, we demonstrate the crucial role of the transcription factor $\Delta \mathrm{Np} 63$ in controlling the enhancer landscape of genes important for the maintenance of lung stem cell populations. Through this regulation, $\triangle \mathrm{Np} 63$ exerts its oncogenic functions in both lung squamous cell carcinoma (LUSC) and adenocarcinoma (LUAD). Importantly, our findings demonstrate that $\triangle \mathrm{Np} 63$ regulates a set of common genes, including $B C L 9 L$, through recruitment of $\triangle \mathrm{Np} 63$ along with histone acetyltransferases p300 to enhancer regions, in both LUSC and LUAD.

Our data are in line with previous reports showing that $\triangle \mathrm{Np} 63$ is required for the maintenance of basal cells in the epidermis ${ }^{12}$, where $\Delta \mathrm{Np} 63$ regulates enhancer-associated genes essential for the homoeostasis ${ }^{48}$ and differentiation ${ }^{49}$ of the epidermis. Notably, the regulation of the enhancer landscape by $\Delta \mathrm{Np} 63$ has also been found in epithelial tumours, including pancreatic adenocarcinomas, where the $\Delta \mathrm{Np} 63$ controlled enhancer-associated genes drive squamous trans-differentiation ${ }^{50}$. Given the commonalities across squamous cell carcinomas of different tissues including LUSC ${ }^{51}$, it is not surprising that analysis of LUSCs by the TCGA showed that $\triangle \mathrm{Np} 63$ is overexpressed in almost half of all these primary tumours ${ }^{19}$. Conversely, until now, the involvement of $\Delta \mathrm{Np} 63$ in the distal lung mainly relied on the findings that transiently amplified distal lung stem cells, which arise after lung injury, are positive for $\mathrm{p} 63^{21,52}$. We now demonstrate a novel mechanism for $\triangle \mathrm{Np} 63$ in AT2 cells, consisting of the upregulation of AT2 cell-specific genes through the $\triangle$ Np63-specific modulation of their enhancer landscape. Our LUAD mouse model obtained by combining the deletion of $\triangle N p 63$ with the $\mathrm{Kras}^{\mathrm{G} 12 \mathrm{D} /+}$ mutation allowed us to demonstrate the tumour initiating role of $\Delta \mathrm{Np} 63$, which is achieved through the regulation of stem cell signalling pathways that are often co-opted in cancer. Indeed, our findings suggest that $\Delta \mathrm{Np} 63$ plays a critical role in the initiation and progression of cancers that are derived from stem cells requiring $\triangle \mathrm{Np} 63$, including the lung basal cells and AT2 cells for LUSC and LUAD, respectively.

By performing ChIP-seq experiments in mouse lung stem cells and non-small cell lung tumours and then validating our findings in human systems, including the TCGA LUSC ${ }^{19}$ and LUAD ${ }^{44}$ datasets, lung cancer xenografts, as well as an alternative exogenous model utilising dCas 9 technology $y^{53}$, we identified a group of enhancer-associated genes regulated by $\triangle \mathrm{Np} 63$ in concert with p300. Among the genes controlled in both LUSC and LUAD, we found $B C L 9 L$, a member of the Wnt pathway ${ }^{54}$ with known oncogenic activities in multiple cancer types, including colon adenocarcinomas ${ }^{23}$, hepatocellular carcinomas ${ }^{24}$ and pancreatic adenocarcinomas ${ }^{25}$. We now show that BCL9L is regulated by $\triangle \mathrm{Np63}$ and acts as a crucial unifying oncogene in non-small cell lung cancers as well. Indeed, not only do high levels of $B C L 9 L$ correlate with poor overall survival in two independent cohorts of LUAD, but the overexpression of BCL9L can also rescue the reduced oncogenic growth caused by the downregulation of $\triangle N p 63$, thus indicating that BCL9L is an important common mediator of the oncogenic activities of $\triangle \mathrm{Np} 63$ in both LUAD and LUSC.

In conclusion, our results demonstrate a unifying oncogenic role for $\Delta \mathrm{Np} 63$ in non-small cell lung cancer consisting of the modulation of the enhancer landscape in lung cancer stem cells. This modulation ultimately leads to the expression of key drivers of LUSC and LUAD, whose inhibition may represent a novel therapeutic approach to treat these highly deadly tumour types.

\section{Methods}

Cell lines and culture conditions. The Kras ${ }^{G 12 D /+}$ expressing human lung cancer cell lines (H520, H2170, H358, H1944 and H2009) were cultured in RPMI medium supplemented with $1 \%$ L-glutamine, $1 \%$ penicillin/streptomycin, and $10 \%$ foetal bovine serum. $293 \mathrm{~T}$ cells were cultured in DMEM medium supplemented with $1 \%$ L-glutamine, $1 \%$ penicillin/streptomycin, and $10 \%$ foetal bovine serum. All the cell lines were authenticated by STR profiling by the MD Anderson Cell Line Authentication Service.

Mouse models. $\Delta N p 63^{f l / f l}$ mice ${ }^{12}$, Rosa $^{M / M}$ mice $^{30}$ and $K r a L S L-G 12 D /+$ mice $^{22}$ were in a C57BL/6 background and were crossed to obtain the following three mouse strains: (i) $\Delta N p 63^{f l / f l} ; \operatorname{Rosa}^{M / M}$, (ii) $\operatorname{Kras}^{G 12 D /+} ; R o s a^{M / M}$, (iii) $\Delta N p 63^{f l / f l}$; $\mathrm{Kras}^{\mathrm{G12D/+}} ; \mathrm{Rosa}^{M / M}$. Mice were aged until $6-8$ weeks old before being utilised in experiments. Both male and female mice were used at equal ratios. Littermates were randomly assigned to experimental groups. Mice were housed pathogen-free and ventilated cages and allowed free access to irradiated food and autoclaved water ad libitum in a $12 \mathrm{~h} \mathrm{light/dark} \mathrm{cycle,} \mathrm{with} \mathrm{room} \mathrm{temperature} \mathrm{at} 21 \pm 2{ }^{\circ} \mathrm{C}$ and humidity between 45 and $65 \%$. All studies were performed in accordance with established protocols approved by the Institutional Animal Care and Use Committee of MD Anderson Cancer Center and H. Lee Moffitt Cancer Center.

Intratracheal infection of mice. Six to eight weeks old mice were anaesthetised with a ketamine/xylazine solution (ketamine $100 \mathrm{mg} / \mathrm{kg} \mathrm{IP,} \mathrm{xylazine} 10 \mathrm{mg} / \mathrm{kg} \mathrm{IP}$ ), intubated using a fibre optic illuminated catheter ${ }^{29}$, and given $2.5 \times 10^{7} \mathrm{PFU}$ viral dosage suspended in $100 \mu \mathrm{l}$ of minimal essential media (MEM). CMV-cre and CMV-empty adenovirus was obtained from the Viral Core at the Baylor College of Medicine. At the indicated timepoints, lungs and tracheas were isolated from euthanized mice and preserved in $10 \%$ formaldehyde for $24 \mathrm{~h}$, followed by pre servation in $70 \%$ ethanol for $24 \mathrm{~h}$. The tissues were then processed by the MD Anderson Research Histology Core Lab.

Immunofluorescence and immunohistochemistry. IF and IHC was performed overnight keeping the slides in humified chambers ${ }^{11}$ and incubating them with the following primary antibodies: $\triangle \mathrm{Np63}$ (ab172731, Abcam, 1:500), GFP (ab13970, Abcam, 1:200), Ki67 (ab15580, Abcam, 1:1,000), cytokeratin 5 (ab53121, Abcam, 1:250), acetylated tubulin (T7451, Sigma-Aldrich, 1:200), mucin 5ac (MA1-21907, Thermo Fisher Scientific, 1:100), cleaved caspase 3 (9661, Cell Signaling, 1:200), CC10 (sc-25555, Santa Cruz, 1:200), SPC (sc-7705, Santa Cruz, 1:100) and NGFR (ab8874, Abcam, 1:100).

Microscopy and image processing. Bright-field and immunofluorescent images were taken on an Olympus IX83 microscope. Immunohistochemical and immunofluorescence images were quantified using ImageJ cell counting software.

Scoring of tracheal sections. Three H\&E sections per mouse were analysed using the Olympus Cellsense software. Tracheal epithelial height was measured from the basement membrane to the apex of the membrane. Epithelial separation (ES) was defined as the length of epithelium where the basement membrane was separated. 
Lung tumour grading. H\&E sections of mouse lungs were examined, and lung lesions were graded $^{22}$ into atypical adenomatous hyperplasia (AAH), grade 1, and grade $2+$. For each mouse, three H\&E sections of five lung lobes taken $100 \mu \mathrm{m}$ apart were analysed.

Polidocanol administration. Fifty microlitres of $2 \%$ polidocanol/PBS was administered to the mouse trachea by a catheter inserted into the trachea ${ }^{34}$.

Mouse tumour xenografts. About $5 \times 10^{6}$ human lung cancer cells were mixed in 1:1 ratio with growth factor reduced matrigel (Corning) in a total volume of $200 \mu \mathrm{l}$ to inject per flank in 6-8 weeks old male athymic $n u / n u$ mice. Tumours were measured weekly with callipers until the final volume reached around $2 \mathrm{~cm}^{3}$. Mice were euthanized and the tumours were excised, measured, fixed in $10 \%$ formaldehyde and submitted for histological processing.

Soft agar colony assay of tumour cell lines. Human lung cancer cells were transfected with siRNAs for $\triangle \mathrm{Np} 63^{16,55}$ and pCMV6-Myc tagged BCL9L (RC218806, OriGene Technologies). siNon-Targeting (siC001, Sigma) and pCMV6 (PS100001, OriGene Technologies) were used as negative controls, respectively. After $24 \mathrm{~h}$, the cells were trypsinized and resuspended in a top layer of their culture medium with $0.3 \%$ agarose (BP160, Fisher) at $2 \times 10^{5}$ cells per well in triplicate in six-well plates and plated on a bottom layer of culture medium containing $1 \%$ agarose. The medium was changed every 2 days. After 3 weeks the colonies were counted with a $20 \mathrm{X}$ objective on a Zeiss Observer.Z1 microscope.

Western blot assay. Human cancer cells were lysed and western blot was performed ${ }^{16}$ using the following primary antibodies were used: $\triangle \mathrm{Np} 63$, (619002, BioLegend, 1:500), BCL9L (PA5-61946, Thermo Fisher, 1:1000), Myc-tag (2276, Cell Signaling, 1:2000) and Actin (4967 S, Cell Signaling, 1:7500).

Isolation of mouse basal stem cells. The tracheas from $\Delta N p 63^{f l / f l} ; R o s a^{M / M}$ mice were isolated and digested with papain at $37^{\circ} \mathrm{C}^{3}$. Basal cells were then cultured on mitomycin treated J2-3T3 feeders for 7 days in MTEC media with rock inhibitor (ab120129, Abcam)

In vitro adenoviral infection of mouse lung cells. Cells were infected at a multiplicity of infection (MOI) of 100. Adenovirus (adeno-empty and adeno-cre) was added to cells in a total volume of $2 \mathrm{ml}$ and incubated at $37^{\circ} \mathrm{C}$ for $1 \mathrm{~h}$, agitating every $15 \mathrm{~min}$. Cells were then washed $2 \mathrm{x}$ with PBS.

Proliferation assay of lung basal stem cell. Basal cells were plated on a bed of mitomycin treated J2-3T3 feeders. 5-ethynyl-2'-deoxyuridine (EdU) was added to the media for $24 \mathrm{~h}$. Cells were fixed with paraformaldehyde and developed using the Click-it EdU Alexa Fluor 488 imaging kit (Invitrogen). Data were analysed on the Nexcelom Celigo.

Sphere formation assay of basal stem cells. Basal cells in single-cell suspension were seeded into $100 \mu \mathrm{l}$ of 1:1 growth factor reduced matrigel:MTEC medium in a 96-well plate. About $100 \mu \mathrm{l}$ of MTEC plus medium was seeded on top of the matrigel. The medium was changed every 2 days. Cells were grown for 1 week. Matrigel was dissolved with cell recovery solution (Corning) and the plate was placed at $4{ }^{\circ} \mathrm{C}$ for $2 \mathrm{~h}$ to allow the spheres to sink to the bottom of the plate. The plate was then scanned and analysed on the Nexcelom Celigo.

Differentiation assay of basal stem cells. Basal cells were seeded into 1:1 GFR matrigel: MTEC plus medium into a 24 well plate insert. MTEC plus medium in the well was changed every 2 days and rock inhibitor was omitted after day 4 to allow for differentiation. After 20 days of growth, the medium was removed and replaced with $4 \%$ paraformaldehyde for $24 \mathrm{~h}$ of fixation. The fixed matrigel sample was removed and embedded in Histogel, followed by histological processing.

Apoptosis assay of basal stem cells. Basal cells were plated on top of mitomycin treated J2-3T3 feeders. Using the Annexin V/Dead Cell Apoptosis Kit (Thermo Fisher), cells were incubated with reagent and subsequently fixed. Analysis was conducted using the Nexcelom Celigo for imaging.

Isolation of distal lung stem cells. BASCs and AT2 cells were isolated from $\Delta N p 3^{f l / f l} ; R o s a^{M / M}$ mice $^{6,56}$. After digestion with collagenase and dispase, cells were stained with fluorescent antibodies: CD31-APC (551262, BD), CD45-APC (559864, BD), EpCAM-PE-Cy7 (118216, BioLegend), SCA-1-APC-Cy7 (560654, $\mathrm{BD})$. Cells were sorted in a MoFlo Astrios cell sorter. AT2 cells are contained in the $\mathrm{CD} 31^{\text {neg, }}$ CD $45^{\text {neg }}$, EpCAMpos and Sca- $1^{\text {neg }}$ population, and BASCs in the $\mathrm{CD} 31^{\text {neg, }}$ CD45 ${ }^{\text {neg, }}$ EpCAMpos and Sca-1 ${ }^{\text {pos }}$ population $^{57}$.

Colony formation assay of distal lung stem cells. BASC and AT2 cells were plated in a gelatin-coated 96 -well plate mixed with primary mouse lung endothelial cells in a ratio of 1000 stem cells to $2 \times 10^{6}$ endothelial cells per plate. The medium was changed every 2 days. The plate was analysed on the Nexcelom Celigo after 1 week of culture ${ }^{6}$ using the colony counting algorithm.

RNA isolation. RNA was extracted from a minimum of $1 \times 10^{6}$ primary cell culture or cancer cell line culture using trizol extraction and the Pure Link RNA mini kit (Ambion).

RNA sequencing. Approximately $5 \mu \mathrm{g}$ of polyA+RNA was used to construct RNA-Seq libraries using the standard Illumina protocol. Mouse and human mRNA sequencing yielded $20-40$ million read pairs for each sample. The mouse mRNASeq reads were mapped using TopHat v2.0.12 onto the mouse genome mm10 (GRCm38) assembly. Then, mapped reads were assembled using cufflinks v2.2.1 to calculate the fragments per kilobase of transcript per million mapped reads (FPKM). A combined profile of all samples was computed. Principal component analysis was executed using the implementation within the $\mathrm{R}$ statistical analysis system. Hierarchical clustering of samples was executed by first computing the symmetrical sample distance matrix using the Pearson correlation between mRNA profiles as a metric, supervised sample analysis was performed using the $t$-test statistics, and heatmaps were generated using the heatmap. 2 package in R. Enriched pathways were determined using the hypergeometric distribution, with significance achieved for FDR-adjusted $P<0.05$, and are reported in the Supplementary dataset 1 .

Low cell number ChIP-sequencing. $\Delta N p 63^{f l f l}$ and $\Delta N p 63^{\Delta / \Delta}$ cells were pelleted and snap-frozen using liquid nitrogen. Low cell number ChIP-seq for H3K27ac (39193, Active Motif), RNA polymerase 2 (39097, Active Motif), and $\Delta$ Np63 (sc8609, Santa Cruz) was performed by Active Motif. Sequencing was analysed with MACS $^{58}$ to call peaks with $q<0.05$ and mapped using bowtie2 against the mouse genome build UCSC mm10. ChIP-Seq signal maps were generated using BEDTools $^{59}$ and visualised using the Integrative Genome Viewer (IGV) ${ }^{60}$. Enhancer candidates were determined using HOMER ${ }^{61}$. Enriched motifs at peaks were determined using HOMER ${ }^{61}$. ChIP-Seq signals at top 2000 enhancer candidates plotted as heatmaps using deepTools ${ }^{62}$ scaled for enhancer length and summaries over all enhancer candidates were computed as average values over all enhancer candidates and plotted. Overlap with known enhancers was determined using BEDTools ${ }^{59}$ against the Fantom 5 enhancer catalogue ${ }^{41}$ and the Mouse ENCODE lung enhancers ${ }^{42}$, and it is reported in Supplementary dataset 3 . The enhancer-associated genes are listed in Supplementary datasets 2 and 4.

For the validation of the selected genes, cellular proteins were cross-linked to DNA using $1 \%$ formaldehyde and chromatin was isolated and sonicated in ChIP buffer $(20 \mathrm{mM}$ Tris- $\mathrm{HCl} \mathrm{pH}$ 7.5, $100 \mathrm{mM} \mathrm{NaCl}, 1 \mathrm{mM}$ EDTA, 0.5\% NP40, 0.5\% sodium deoxycholate, $0.1 \% \mathrm{SDS})^{63}$. Each ChIP was performed in triplicate using $2 \mu \mathrm{g}$ of either H3K27ac (39193, Active Motif), RNA polymerase 2 (39097, Active Motif) or $\Delta \mathrm{Np} 63$ (sc-8609, Santa Cruz). As negative controls for the immunoprecipitation, IgG purified from mouse serum (sc-2025, Santa Cruz) and rabbit serum (sc-2027, Santa Cruz) were used. The presence of H3K27ac, RNA polymerase 2 and $\triangle \mathrm{Np} 63$ was analysed by qRT-PCR with the primers listed in Supplementary dataset 7

Quantitative real time PCR. Total RNA was prepared using TRIzol reagent (Invitrogen). For gene expression analysis, complementary DNA was synthesised from $5 \mu \mathrm{g}$ of total RNA using the SuperScript II First-Strand Synthesis Kit (Invitrogen) according to the manufacturer's protocol followed by qRT-PCR using BioRad SYBR green master mix. The qRT-PCR data were analysed using the $\Delta \Delta \mathrm{CT}$ analysis. The utilised primers are listed in Supplementary dataset 8 .

dCas9 assay. Experiments were performed in $293 \mathrm{~T}$ cells as previously reported ${ }^{53}$ Briefly, four sgRNA per genomic site were designed using the Broad sgRNA design tool and cloned into the pLX-sgRNA vector. Plasmids for dCas9, dCas9-p300 and dCas9- $\Delta$ Np63 were utilised in a 3:1 ratio to sgRNA vectors. All plasmids were transfected using Lipofectamine 2000. The cells were incubated for $48 \mathrm{~h}$ before collection of the RNA. The utilised primers are listed in Supplementary dataset 9

The Cancer Genome Atlas (TCGA) lung adenocarcinoma (LUAD) and lung squamous cell carcinoma (LUSC) datasets. Clinical, mutation data and normalised RNA-seq expression data (RSEM-Batch normalised from Illumina HiSeq_RNASeqV2) of TCGA LUAD and LUSC patients were obtained from the cBioPortal (https://www.cbioportal.org) pan-cancer cohort. Out of 566 LUAD patients in the dataset, 510 patients have RNA-seq data where 501 patients had complete overall survival information and 508 samples had AJCC pathologic tumour stage information. Similarly, a total of 487 LUSC patients were found in the clinical data, with 484 of those were available in the RNA-seq expression data, and 478 patients with complete overall survival information availability were used for overall survival analysis.

Moffitt LUAD and LUSC datasets. For the Moffitt LUAD cohort, gene expression profiles of 442 patients were downloaded from GEO (GSE72094). This dataset was 
profiled by the Rosetta/Merck Human RSTA Custom Affymetrix 2.0 microarray and the data were normalised by IRON as previously described ${ }^{64}$. About 398 patients have the survival information and were used for the survival analysis. For the Moffitt LUSC cohort, 116 patients were profiled by RNA-seq and 108 had clinical outcome data as previously described ${ }^{65}$.

Overall survival analysis. Median gene expression of BCL9L was used as the cutpoint to classify high and low expressed groups. Kaplan-Meier overall survival analysis was performed between the high and low gene expression group using 'KapalanMeierFitter' function in 'lifelines' Python package (version 0.25.4). P values were obtained using log rank test through 'multivariate_logrank_test' function in the same package and $P<0.05$ was considered statistically significant in this analysis.

Statistical analyses and reproducibility. All the experiments are representative of at least three independent replicates. Data collection was performed with Microsoft Excel and data analysis was performed using GraphPad Prism. Gels, blots and images are representative of three independent experiments giving similar results.

Reporting summary. Further information on research design is available in the Nature Research Reporting Summary linked to this article.

\section{Data availability}

Publicly available data used in this paper were obtained from UCSC (http:// genome.ucsc.edu/cgibin/hgGateway) and The Cancer Genome Atlas (TCGA) lung adenocarcinoma dataset (http://www.cancer.gov/about-nci/organization/ccg/research/ structural-genomics/tcga). The RNA-seq data and the ChIP-seq data were deposited to NCBI Gene Expression Omnibus (GEO) repository (series GSE131671). All the imaging data supporting the findings of this study are available from the corresponding authors upon reasonable request. The source data for all the other results are provided as Source Data file with this paper. Source data are provided with this paper.

Received: 11 March 2021; Accepted: 11 January 2022;

Published online: 01 February 2022

\section{References}

1. Reya, T., Morrison, S. J., Clarke, M. F. \& Weissman, I. L. Stem cells, cancer, and cancer stem cells. Nature 414, 105-111 (2001).

2. Eramo, A., Haas, T. L. \& De Maria, R. Lung cancer stem cells: tools and targets to fight lung cancer. Oncogene 29, 4625-4635 (2010).

3. Rock, J. R. et al. Basal cells as stem cells of the mouse trachea and human airway epithelium. Proc. Natl Acad. Sci. USA 106, 12771-12775 (2009).

4. Hanna, J. M. \& Onaitis, M. W. Cell of origin of lung cancer. J. Carcinog. 12, 6 (2013).

5. $\mathrm{Xu}, \mathrm{X}$. et al. Evidence for type II cells as cells of origin of K-Ras-induced distal lung adenocarcinoma. Proc. Natl Acad. Sci. USA 109, 4910-4915 (2012).

6. Kim, C. F. et al. Identification of bronchioalveolar stem cells in normal lung and lung cancer. Cell 121, 823-835 (2005)

7. Wansleeben, C., Barkauskas, C. E., Rock, J. R. \& Hogan, B. L. Stem cells of the adult lung: their development and role in homeostasis, regeneration, and disease. Wiley Interdiscip. Rev. Dev. Biol. 2, 131-148 (2013).

8. Su, X., Chakravarti, D. \& Flores, E. R. p63 steps into the limelight: crucial roles in the suppression of tumorigenesis and metastasis. Nat. Rev. Cancer 13, 136-143 (2013).

9. Yang, A. et al. p63 is essential for regenerative proliferation in limb, craniofacial and epithelial development. Nature 398, 714-718 (1999).

10. Mills, A. A. et al. p63 is a p53 homologue required for limb and epidermal morphogenesis. Nature 398, 708-713 (1999).

11. Su, X. et al. TAp63 prevents premature aging by promoting adult stem cell maintenance. Cell Stem Cell 5, 64-75 (2009).

12. Chakravarti, D. et al. Induced multipotency in adult keratinocytes through down-regulation of DeltaNp63 or DGCR8. Proc. Natl Acad. Sci. USA 111, E572-E581 (2014).

13. Abbas, H. A. et al. Distinct TP63 isoform-driven transcriptional signatures predict tumor progression and clinical outcomes. Cancer Res. 78, 451-462 (2018).

14. Su, X. et al. TAp63 suppresses metastasis through coordinate regulation of Dicer and miRNAs. Nature 467, 986-990 (2010).

15. Venkatanarayan, A. et al. IAPP-driven metabolic reprogramming induces regression of p53-deficient tumours in vivo. Nature 517, 626-630 (2015).

16. Napoli, M. et al. $\triangle$ Np63/DGCR8-dependent microRNAs mediate therapeutic efficacy of HDAC inhibitors in cancer. Cancer Cell 29, 874-888 (2016).
17. Venkatanarayan, A., Raulji, P., Norton, W. \& Flores, E. R. Novel therapeutic interventions for p53-altered tumors through manipulation of its family members, p63 and p73. Cell Cycle 15, 164-171 (2016).

18. Conde, E. et al. The use of P63 immunohistochemistry for the identification of squamous cell carcinoma of the lung. PloS one 5, e12209 (2010).

19. Cancer Genome Atlas Research, N. Comprehensive genomic characterization of squamous cell lung cancers. Nature 489, 519-525 (2012)

20. Yan, W., Wistuba, I. I., Emmert-Buck, M. R. \& Erickson, H. S. Squamous cell carcinoma - Similarities and differences among anatomical sites. Am. J. Cancer Res. 1, 275-300 (2011).

21. Zuo, W. et al. p63(+)Krt5(+) distal airway stem cells are essential for lung regeneration. Nature 517, 616-620 (2015).

22. Jackson, E. L. et al. Analysis of lung tumor initiation and progression using conditional expression of oncogenic K-ras. Genes Dev. 15, 3243-3248 (2001).

23. Gay, D. M. et al. Loss of BCL9/9l suppresses Wnt driven tumourigenesis in models that recapitulate human cancer. Nat. Commun. 10, 723 (2019).

24. Huge, N. et al. Wnt status-dependent oncogenic role of BCL9 and BCL9L in hepatocellular carcinoma. Hepatol. Int. 14, 373-384 (2020).

25. Sannino, G. et al. Role of BCL9L in transforming growth factor-beta (TGFbeta)-induced epithelial-to-mesenchymal-transition (EMT) and metastasis of pancreatic cancer. Oncotarget 7, 73725-73738 (2016).

26. Daniely, Y. et al. Critical role of p63 in the development of a normal esophageal and tracheobronchial epithelium. Am. J. Physiol. Cell Physiol. 287, C171-C181 (2004).

27. Arason, A. J. et al. deltaNp63 has a role in maintaining epithelial integrity in airway epithelium. PLoS ONE 9, e88683 (2014).

28. Nonaka, D. A study of DeltaNp63 expression in lung non-small cell carcinomas. Am. J. Surg. Pathol. 36, 895-899 (2012).

29. DuPage, M., Dooley, A. L. \& Jacks, T. Conditional mouse lung cancer models using adenoviral or lentiviral delivery of Cre recombinase. Nat. Protoc. 4, 1064-1072 (2009).

30. Muzumdar, M. D., Tasic, B., Miyamichi, K., Li, L. \& Luo, L. A global doublefluorescent Cre reporter mouse. Genesis 45, 593-605 (2007).

31. Koster, M. I. \& Roop, D. R. The role of p63 in development and differentiation of the epidermis. J. Dermatol. Sci. 34, 3-9 (2004).

32. Carroll, D. K. et al. p63 regulates an adhesion programme and cell survival in epithelial cells. Nat. Cell Biol. 8, 551-561 (2006).

33. Jeong, Y. et al. Role of KEAP1/NRF2 and TP53 mutations in lung squamous cell carcinoma development and radiation resistance. Cancer Disco. 7, 86-101 (2017).

34. Paul, M. K. et al. Dynamic changes in intracellular ROS levels regulate airway basal stem cell homeostasis through Nrf2-dependent Notch signaling. Cell Stem Cell 15, 199-214 (2014)

35. Pott, S. \& Lieb, J. D. What are super-enhancers? Nat. Genet 47, 8-12 (2015).

36. Sur, I. \& Taipale, J. The role of enhancers in cancer. Nat. Rev. Cancer 16, 483-493 (2016)

37. Whyte, W. A. et al. Master transcription factors and mediator establish superenhancers at key cell identity genes. Cell 153, 307-319 (2013).

38. Hnisz, D. et al. Super-enhancers in the control of cell identity and disease. Cell 155, 934-947 (2013).

39. Loven, J. et al. Selective inhibition of tumor oncogenes by disruption of superenhancers. Cell 153, 320-334 (2013)

40. Fornes, O. et al. JASPAR 2020: update of the open-access database of transcription factor binding profiles. Nucleic Acids Res. 48, D87-D92 (2020).

41. Abugessaisa, I. et al. FANTOM enters 20th year: expansion of transcriptomic atlases and functional annotation of non-coding RNAs. Nucleic Acids Res. 49, D892-D898 (2021)

42. Shen, Y. et al. A map of the cis-regulatory sequences in the mouse genome. Nature 488, 116-120 (2012)

43. Zhang, Z. et al. Transcription factor Etv5 is essential for the maintenance of alveolar type II cells. Proc. Natl Acad. Sci. USA 114, 3903-3908 (2017).

44. Cancer Genome Atlas Research, N. Comprehensive molecular profiling of lung adenocarcinoma. Nature 511, 543-550 (2014).

45. Hilton, I. B. et al. Epigenome editing by a CRISPR-Cas9-based acetyltransferase activates genes from promoters and enhancers. Nat. Biotechnol. 33, 510-517 (2015).

46. Visel, A., Rubin, E. M. \& Pennacchio, L. A. Genomic views of distant-acting enhancers. Nature 461, 199-205 (2009).

47. Feinberg, A. P., Koldobskiy, M. A. \& Gondor, A. Epigenetic modulators, modifiers and mediators in cancer aetiology and progression. Nat. Rev. Genet 17, 284-299 (2016)

48. Lin-Shiao, E. et al. KMT2D regulates p63 target enhancers to coordinate epithelial homeostasis. Genes Dev. 32, 181-193 (2018).

49. Kouwenhoven, E. N. et al. Transcription factor p63 bookmarks and regulates dynamic enhancers during epidermal differentiation. EMBO Rep. 16, 863-878 (2015). 
50. Somerville, T. D. D. et al. TP63-mediated enhancer reprogramming drives the squamous subtype of pancreatic ductal adenocarcinoma. Cell Rep. 25, 1741-1755 e1747 (2018).

51. Hoadley, K. A. et al. Multiplatform analysis of 12 cancer types reveals molecular classification within and across tissues of origin. Cell 158, 929-944 (2014).

52. Zhao, R. et al. Yap tunes airway epithelial size and architecture by regulating the identity, maintenance, and self-renewal of stem cells. Dev. Cell 30, 151-165 (2014).

53. Wang, S. P. et al. A UTX-MLL4-p300 transcriptional regulatory network coordinately shapes active enhancer landscapes for eliciting transcription. Mol. Cell 67, 308-321 e306 (2017).

54. Katoh, M. \& Katoh, M. WNT signaling pathway and stem cell signaling network. Clin. Cancer Res. 13, 4042-4045 (2007).

55. Bui, N. H. B. et al. Spatiotemporal regulation of DeltaNp63 by TGFbetaregulated miRNAs is essential for cancer metastasis. Cancer Res. 80, 2833-2847 (2020).

56. Driscoll, B. et al. Isolation and characterization of distal lung progenitor cells. Methods Mol. Biol. 879, 109-122 (2012).

57. Bortnick, A. E. et al. Identification and characterization of rodent ABCA1 in isolated type II pneumocytes. Am. J. Physiol. Lung Cell Mol. Physiol. 285, L869-L878 (2003).

58. Zhang, Y. et al. Model-based analysis of ChIP-Seq (MACS). Genome Biol. 9, R137 (2008).

59. Quinlan, A. R. \& Hall, I. M. BEDTools: a flexible suite of utilities for comparing genomic features. Bioinformatics 26, 841-842 (2010).

60. Thorvaldsdottir, H., Robinson, J. T. \& Mesirov, J. P. Integrative genomics viewer (IGV): high-performance genomics data visualization and exploration. Brief. Bioinform. 14, 178-192 (2013).

61. Heinz, S. et al. Simple combinations of lineage-determining transcription factors prime cis-regulatory elements required for macrophage and B cell identities. Mol. Cell 38, 576-589 (2010).

62. Ramirez, F., Dundar, F., Diehl, S., Gruning, B. A. \& Manke, T. deepTools: a flexible platform for exploring deep-sequencing data. Nucleic Acids Res. 42, W187-W191 (2014).

63. Napoli, M. et al. Pan-cancer analysis reveals TAp63-regulated oncogenic IncRNAs that promote cancer progression through AKT activation. Nat. Commun. 11, 5156 (2020).

64. Schabath, M. B. et al. Differential association of STK11 and TP53 with KRAS mutation-associated gene expression, proliferation and immune surveillance in lung adenocarcinoma. Oncogene 35, 3209-3216 (2016).

65. Stewart, P. A. et al. Proteogenomic landscape of squamous cell lung cancer Nat. Commun. 10, 3578 (2019).

\section{Acknowledgements}

We thank C. Kim and M. Paschini for generously sharing protocols and cell lines for AT2 and BASC isolation and culture. We thank B. Gomperts for sharing polidocanol protocols. We thank C. Liu and J. Kurie for intratracheal instillation training and P. Raulji for assistance with the maintenance of mouse colonies. This work was supported by R35CA197452 to ERF, R01 CA207098, and R01 CA207109 to MGL, and CPRIT RP140271 to ERF and MGL. ERF is a National Cancer Institute Outstanding Investi- gator, Moffitt Distinguished Scholar, and Scholar of the Leukaemia and Lymphoma Society, the Rita Allen Foundation, and the V Foundation for Cancer Research. MN is a Scholar of the Cancer Prevention Research Institute of Texas-Translational Research in Multidisciplinary Programme. SJW was supported by T32 in Molecular Genetics of Cancer (T32 CA009299), the Schissler Foundation for Translational Studies of Common Human Diseases, and the Andrew Sowell-Wade Huggins Scholarship in Cancer Research. KR and CC have been supported in part by NIH P30 shared resource grant CA125123, CPRIT RP200504, NIEHS P30 Center grant ES030285, and NIEHS P42 ES027725. KL was supported by T32 in Integrated Programme in Cancer and Data Science (T32 CA233399).

\section{Author contributions}

M.N., S.J.W. and E.R.F. conceived the study, designed experiments and analysed data. M.N., S.J.W., B.L.G., R.C. and S.D. performed experiments. C.C., H.A.A., K.L., K.R. and A.C.T. conducted bioinformatics analyses. M.N., S.J.W., M.G.L. and E.R.F. wrote the paper. All authors discussed the paper and commented on the manuscript.

\section{Competing interests}

The authors declare no competing interests.

\section{Additional information}

Supplementary information The online version contains supplementary material available at https://doi.org/10.1038/s41467-022-28202-1.

Correspondence and requests for materials should be addressed to Min Gyu Lee, Cristian Coarfa or Elsa R. Flores.

Peer review information Nature Communications thanks De-chen Lin, Giovanni Blandino and the other anonymous reviewer(s) for their contribution to the peer review this work. Peer reviewer reports are available.

Reprints and permission information is available at http://www.nature.com/reprints

Publisher's note Springer Nature remains neutral with regard to jurisdictional claims in published maps and institutional affiliations.

Open Access This article is licensed under a Creative Commons Attribution 4.0 International License, which permits use, sharing, adaptation, distribution and reproduction in any medium or format, as long as you give appropriate credit to the original author(s) and the source, provide a link to the Creative Commons license, and indicate if changes were made. The images or other third party material in this article are included in the article's Creative Commons license, unless indicated otherwise in a credit line to the material. If material is not included in the article's Creative Commons license and your intended use is not permitted by statutory regulation or exceeds the permitted use, you will need to obtain permission directly from the copyright holder. To view a copy of this license, visit http://creativecommons.org/ licenses/by/4.0\%.

(C) The Author(s) 2022, corrected publication 2022 